\title{
Divided Differences, Square Functions and a Law of the Iterated Logarithm
}

\author{
Artur Nicolau \\ Departament de Matemàtiques \\ Universitat Autònoma de Barcelona \\ 08193 Barcelona, Spain \\ artur@mat.uab.es
}

\section{Introduction}

Let $f$ be a real valued measurable function defined in an open set $\mathcal{U} \subset \mathbb{R}^{d}$. If $x, t \in \mathbb{R}^{d}$ satisfy $x-t, x, x+t \in \mathcal{U}$, we consider the (symmetric) divided difference $\Delta(f)(x, t)$ and the second (symmteric) divided difference $\Delta_{2}(f)(x, t)$ defined as

$$
\begin{aligned}
\Delta(f)(x, t) & =\frac{f(x+t)-f(x-t)}{2|t|}, \\
\Delta_{2}(f)(x, t) & =\frac{f(x+t)+f(x-t)-2 f(x)}{2|t|} .
\end{aligned}
$$

It is well known that differentiability properties of the function $f$ can be described by size conditions on the differences $\Delta_{2} f$. Actually for $\delta>0$ consider the square function

$$
g_{\delta}^{2}(f)(x)=\int_{\|t\|<\delta} \Delta_{2}^{2}(f)(x, t) \frac{d m(t)}{|t|^{d}}, \quad x \in \mathcal{U}
$$

where $d m(t)$ denotes Lebesgue measure in $\mathbb{R}^{d}$. We denote $g(f)=g_{1}(f)$. A classical result by Stein and Zygmund, extending previous work by Marcinkiewicz and Zygmund, says that the set of points in $\mathcal{U}$ where $f$ is differentiable and the set of points $x \in \mathcal{U}$ for which there exists $\delta=\delta(x)>0$ such that $g_{\delta}(f)(x)<\infty$ and $\sup \left\{\left|\Delta_{2} f(x, h)\right|:\|h\|<\delta\right\}<\infty$, can differ at most by a set of Lebesgue measure zero. See [SZ2] or [St1, p. 262].

In this work we study the growth of the divided differences of a function at the points where the function is not differentiable. In the one dimensional case, under certain assumptions on the function, Anderson and Pitt obtained very nice results in their paper [AP]. For instance they considered the Zygmund class of continuous one variable functions $f$ for which $\|f\|_{*}=\sup \left\{\left|\Delta_{2}(f)(x, h)\right|\right.$ : $x, h \in \mathbb{R}\}<\infty$. Since $|\Delta(f)(x+h, h)-\Delta(f)(x+h / 2, h / 2)| \leq\left|\Delta_{2} f(x+h, h)\right|$, for any $x, h \in \mathbb{R}$, iterating one obtains

$$
\left|\frac{f(x+h)-f(x)}{h}\right| \leq\|f\|_{*} \ln _{2}(1 / h)+2\left|f\left(x+2^{N} h\right)-f(x)\right|, x \in \mathbb{R}, 0<h<1 / 2
$$

The author is supported in part by the grants MTM2008-00145 and 2009SGR420 
where $N$ is the integer such that $1 / 2<2^{N} h<1$. Hence for any $x \in \mathbb{R}$, the growth of the divided differences $|\Delta(f)(x, h)|$ is at most proportional to $\ln (1 / h)$ for $0<h<1 / 2$. Moreover this uniform estimate is sharp. However, Anderson and Pitt proved the following pointwise estimate which is a version of Kolmogorov's Law of the Iterated Logarithm and improves the previous trivial estimate. At almost every point $x \in \mathbb{R}$, one has

$$
\limsup _{h \rightarrow 0} \frac{|f(x+h)-f(x)|}{|h| \sqrt{\ln 1 /|h| \ln \ln \ln 1 /|h|}} \leq C\|f\|_{*},
$$

where $C$ is a universal constant. The result is sharp. For instance, fixed $b>1$, the WeierstrassHardy lacunary series

$$
f_{b}(x)=\sum_{n=1}^{\infty} b^{-n} \cos \left(b^{n} x\right), \quad x \in \mathbb{R}
$$

is in the Zygmund class and there exists a constant $C_{1}=C_{1}(b)$ such that the limsup in (1.1) is bigger than $C_{1}$ at almost every $x \in \mathbb{R}$. See [W]. Differentiability of functions in the Zygmund class has been studied in [Ma], [DLIN1] and [DLIN2]. The result of Anderson and Pitt is very nice but the assumption that $f$ is in the Zygmund class is somewhat unnatural. Also, instead of estimating the divided differences of a function by a logarithm of the scale, one expects to estimate them by truncated versions of convenient square functions. This is what happens when studying boundary behavior of harmonic functions in the upper-half space. Let $u$ be a harmonic function in an upper half space and let $A(u)$ be its Lusin area function. Classical results of Calderón, Zygmund and Stein tell that the set of points where $u$ has non-tangential limit and the set of points where $A(u)$ is finite, can differ at most by a set of Lebesgue measure 0. See for instance [St1, p. 206] or [BM, p. 43]. On the complement of this set, the growth of $u$ is controlled by a truncated variant of $A(u)$ via a convenient version of the Law of the Iterated Logarithm. See [BKM1, [BKM2] or [BM, p. 65].

Let us first restrict attention to the one dimensional case. Let $\mathcal{U}$ be an open set of the real line $\mathbb{R}$ and let $f \in L_{\text {loc }}^{2}(\mathcal{U})$. Given $x \in \mathcal{U}$ consider $h_{0}=h_{0}(x)=\min \{1, \operatorname{dist}(x, \mathbb{R} \backslash \mathcal{U}) / 2\}$. Instead of the vertical square function $g(f)$, consider the conical square function $A(f)$ defined as

$$
A^{2}(f)(x)=\int_{\Gamma(x)} \Delta_{2}^{2}(f)(s, t) \frac{d s d t}{t^{2}}, \quad x \in \mathbb{R},
$$

where $\Gamma(x)=\left\{(s, t) \in \mathbb{R}_{+}^{2}:|s-x|<t<h_{0}\right\}$ is the cone centered at $x$ of height $h_{0}$. In contrast with (1.1), we do not want to assume any kind of regularity on the function $f$. Since the behavior of the divided differences of a function $f$ may change completely if one changes the definition of $f$ in a set of Lebesgue measure zero, one can not expect to control the divided differences by an square function as $A(f)$ or $g(f)$. However, it turns out that means of divided differences defined as

$$
\tilde{\Delta}(f)(x, h)=\int_{h / 2}^{h} \int_{x-t}^{x+t} \Delta(f)(s, t) \frac{d s d t}{2 t^{2}} \quad, x \in \mathbb{R}, 0<h<1,
$$

can be controlled by truncated versions of $A(f)$ defined as

$$
A^{2}(f)(x, h)=\int_{\Gamma(x) \cap\{t \geq h\}} \Delta_{2}^{2}(f)(s, t) \frac{d s d t}{t^{2}}, \quad x \in \mathbb{R}, \quad 0<h<1 .
$$

Theorem 1. Let $f \in L_{\text {loc }}^{2}(\mathcal{U})$. Then at almost every point $x \in\{x \in \mathcal{U}: A(f)(x)=\infty\}$, one has

$$
\limsup _{h \rightarrow 0} \frac{|\tilde{\Delta}(f)(x, h)|}{\sqrt{A^{2}(f)(x, h) \ln \ln A^{2}(f)(x, h)}} \leq \sqrt{2 \ln 2} .
$$


The result is sharp up to the value $\sqrt{2 \ln 2}$ in the sense that when $f=f_{b}$ is the Hardy-Weierstrass lacunary series mentioned above, the limsup in the statement is bounded below at almost every point $x \in \mathbb{R}$. Let $f$ be a function in the Zygmund class. Since there exists an absolute constant $C>0$ such that $|\Delta(f)(x, h)-\tilde{\Delta}(f)(x, h)| \leq C\|f\|_{*}$ and $A^{2}(f)(x, h) \leq C\|f\|_{*}^{2} \ln (1 / h)$, the estimate (1.1) of Anderson and Pitt follows from Theorem 1. It is worth mentioning that we do not know if the analogue of Theorem 1 holds when one replaces $A(f)(x, h)$ by a truncated version of $g_{1}(f)$. An analogue situation occurs when studying the growth of a harmonic function in an upper half space outside its Fatou set. As mentioned above, Bañuelos, Klemes and Moore proved a version of the Law of the Iterated Logarithm which controls the growth of the harmonic function in terms of the size of its truncated area function. See [BKM1] or [BM, p. 65]. However a similar result replacing the conical Lusin area function by the vertical Littlewood-Payley function is not known. See [BM, p. 114].

The main technical step in the proof of our result is the following good $\lambda$-inequality with provides the right subgaussian decay: there exists a universal constant $C>0$ such that for any $f \in L^{2}([0,1])$ and any numbers $N, M>0$, one has

$$
\left|\left\{x \in[0,1]: \sup _{1 \geq y \geq h}(\tilde{\Delta}(f)(x, y)-\tilde{\Delta}(f)(x, 1)) \geq M ; A^{2}(f)(x, h) \leq N\right\}\right| \leq C \exp \left(-M^{2} / C N\right)
$$

Theorem 1 follows from this subgaussian estimate by standard arguments. Subgaussian estimates in different contexts in analysis can be founded in [CWW], BKM1, BM], $\mathrm{Ma}$, and [SV]. Our proof of (1.2) is organized in two steps. First we state and prove a dyadic version of (1.2) and later we use an averaging procedure due to J. Garnett and P. Jones ([GJ]) to transfer the result in the dyadic setting to the continuous one.

The square function $A(f)$ can also be used as a substitute of $g(f)$ in the classical result of Stein and Zygmund mentioned above. More concretely the following analogue of this classical result holds.

Theorem 2. Let $f$ be a measurable function defined in an open set $\mathcal{U} \subset \mathbb{R}$. Consider the set $A=\{x \in \mathcal{U}: f$ is differentiable at $x\}$ and the set $B$ of points $x \in \mathcal{U}$ for which there exists $\delta=\delta(x)>0$ such that $\sup \left\{\left|\Delta_{2}(f)(x, h)\right|:|h|<\delta\right\}<\infty$ and

$$
\int_{\Gamma(x) \cap\{0<t<\delta\}} \Delta_{2}^{2}(f)(s, t) \frac{d s d t}{t^{2}}<\infty .
$$

Then, the sets $A$ and $B$ can differ at most by a set of Lebesgue measure zero.

Observe that if we change the function $f$ at a set of Lebesgue measure zero, the set of points where $f$ is differentiable may change completely but the square function $A(f)$ remains unchanged. So, the condition $\sup \left|\Delta_{2}(f)(x, h)\right|<\infty$ in the set $B$ is really needed.

For $1<p<\infty$ let $W^{1, p}\left(\mathbb{R}^{d}\right)$ be the Sobolev space of functions in $L^{p}\left(\mathbb{R}^{d}\right)$ whose partial derivatives, in the sense of distributions, are in $L^{p}\left(\mathbb{R}^{d}\right)$. If $2 d /(d+1)<p<\infty$, a function $f \in L^{p}\left(\mathbb{R}^{d}\right)$ is in the Sobolev space $W^{1, p}\left(\mathbb{R}^{d}\right)$ if and only if $g_{1}(f) \in L^{p}\left(\mathbb{R}^{d}\right)$. See [St1, p. 163]. Note that when $d=1$, the result holds for any $1<p<\infty$. A similar result holds in our setting.

Theorem 3. Let $1<p<\infty$. A function $f \in L^{p}(\mathbb{R})$ is in the Sobolev space $W^{1, p}(\mathbb{R})$ if and only if $A(f) \in L^{p}(\mathbb{R})$. Moreover, there exists a constant $C=C(p)>0$ such that $C^{-1}\|A(f)\|_{p} \leq\left\|f^{\prime}\right\|_{p} \leq$ $C\|A(f)\|_{p}$ for any $f \in W^{1, p}(\mathbb{R})$.

Let us now explain our results in higher dimensions. We start recalling some classical results. Rademacher's Theorem says that a Lipschitz function defined in an open set of $\mathbb{R}^{d}$ is differentiable 
at almost every point of the open set. A classical refinement due to Stepanov says that a measurable function $f$ defined in an open set $\mathcal{U} \subset \mathbb{R}^{d}$ is differentiable at almost every point of the set

$$
\left\{x \in \mathcal{U}: \limsup _{|h| \rightarrow 0} \frac{|f(x+h)-f(x)|}{|h|}<\infty\right\}
$$

See $\left[\mathrm{St}\right.$, p. 250]. Stepanov also constructed a continuous nowhere differentiable function in $\mathbb{R}^{2}$ whose ordinary partial derivatives exist at almost every point. Fixed $x \in \mathbb{R}^{d}$ and $\varepsilon>0$, consider the condition

$$
\sup _{h \in \mathbb{R}^{d}:|h|<\varepsilon}\left|\Delta_{2}(f)(x, h)\right|<\infty
$$

This condition is certainly satisfied if $f$ is differentiable at the point $x$, but, as mentioned before, the converse is far from being true. It turns out that (1.3) plays the role of a Tauberian condition allowing one to deduce differentiability from existence of partial derivatives. This is the content of next result which may have independent interest. It is analogue to a classical result by Stein and Zygmund where under the assumption (1.3), one deduces ordinary differentiability at almost every point where differentiability in the harmonic sense holds. See [St, p.260].

Lemma 1. Let $\left\{e_{i}: i=1,2, \ldots, d\right\}$ be the canonical basis of $\mathbb{R}^{d}$. Let $f$ be a measurable function defined in an open set $\mathcal{U} \subset \mathbb{R}^{d}$. Then $f$ is differentiable at almost every point $x \in \mathcal{U}$ where the following two conditions hold

$$
\limsup _{t \in \mathbb{R}, t \rightarrow 0}\left|\frac{f\left(x+t e_{i}\right)-f(x)}{t}\right|<\infty, i=1, \ldots, d
$$

and

$$
\limsup _{h \in \mathbb{R}^{d},|h| \rightarrow 0}\left|\Delta_{2}(f)(x, h)\right|<\infty
$$

Let $\mathcal{U}$ be an open set in the euclidean space $\mathbb{R}^{d}$. Let $f \in L_{\text {loc }}^{2}(\mathcal{U})$. Given $\xi \in \mathbb{R}^{d}$ with $|\xi|=1$, for $x \in \mathcal{U}$ and $0<t<h_{0}=\min \left\{1, \operatorname{dist}\left(x, \mathbb{R}^{d} \backslash \mathcal{U}\right) / 2\right\}$, consider the divided difference and the second divided difference in the direction of $\xi$ given by $\Delta_{\xi}(f)(x, t)=(f(x+t \xi)-f(x-t \xi)) / 2 t$ and $\Delta_{2, \xi}(f)(x, t)=(f(x+t \xi)+f(x-t \xi)-2 f(x)) / 2 t$. For $x \in \mathcal{U}$ and $0<h<h_{0}$, the mean divided difference of $f$ in the direction $\xi$ is defined as

$$
\tilde{\Delta}_{\xi}(f)(x, h)=\int_{h / 2}^{h} \int_{-t}^{t} \Delta_{\xi}(f)(x+s \xi, t) \frac{d s d t}{2 t^{2}}
$$

and the square function in the direction $\xi$ is defined as

$$
A_{\xi}^{2}(f)(x, h)=\int_{h}^{h_{0}} \int_{-t}^{t} \Delta_{2, \xi}^{2}(f)(x+s \xi, t) \frac{d s d t}{t^{2}}
$$

Note that both $\tilde{\Delta}_{\xi}(f)(x, h)$ and $A_{\xi}^{2}(f)(x, h)$ are defined at almost every point $x \in \mathcal{U}$. As before, we denote $A_{\xi}(f)(x)=A_{\xi}(f)(x, 0)$. Our one dimensional results easily give the following statement.

Theorem 4. Let $\mathcal{U}$ be an open subset of $\mathbb{R}^{d}$. Fix $\xi \in \mathbb{R}^{d}$ with $|\xi|=1$.

(a) Let $f$ be a measurable function defined in $\mathcal{U}$. Consider the set $A$ of points in $\mathcal{U}$ on which $f$ has directional derivative in the direction of $\xi$ and the set $B$ of points $x \in \mathcal{U}$ for which there exists $\delta=\delta(x)>0$ such that $\sup \left\{\left|\Delta_{2}(f)(x, h)\right|:|h|<\delta\right\}<\infty$ and

$$
\int_{0}^{\delta} \int_{-t}^{t} \Delta_{2, \xi}^{2}(f)(x+s \xi, t) \frac{d s d t}{t^{2}}<\infty
$$


Then, the sets $A$ and $B$ can differ at most by a set of Lebesgue measure zero.

(b) Assume $f \in L_{\text {loc }}^{2}(\mathcal{U})$. At almost every point $x \in\left\{x \in \mathcal{U}: A_{\xi}(f)(x)=\infty\right\}$, one has

$$
\limsup _{h \rightarrow 0} \frac{\left|\tilde{\Delta}_{\xi}(f)(x, h)\right|}{\sqrt{A_{\xi}^{2}(f)(x, h) \ln \ln A_{\xi}^{2}(f)(x, h)}} \leq \sqrt{2 \ln 2} .
$$

(c) Let $1<p<\infty$ and assume $f \in L^{p}\left(\mathbb{R}^{d}\right)$. Then the directional derivative in the sense of distributions $D_{\xi} f$ is a function in $L^{p}\left(\mathbb{R}^{d}\right)$ if and only if $A_{\xi}(f) \in L^{p}\left(\mathbb{R}^{d}\right)$. Moreover there exists a constant $C=C(p, d)>0$ independent of $f$ and $\xi$ such that $C^{-1}\left\|A_{\xi}(f)\right\|_{p} \leq\left\|D_{\xi}(f)\right\|_{p} \leq C\left\|A_{\xi}(f)\right\|_{p}$ for any $f \in L^{p}\left(\mathbb{R}^{d}\right)$ such that $D_{\xi} f \in L^{p}\left(\mathbb{R}^{d}\right)$.

Given distinct points $\xi_{1}, \ldots, \xi_{d}$ in the unit sphere of $\mathbb{R}^{d}$, consider

$$
\mathcal{A}_{\delta}^{2}(f)(x)=\sum_{i=1}^{d} \int_{0}^{\delta} \int_{-t}^{t} \Delta_{2, \xi_{i}}^{2}(f)\left(x+s \xi_{i}, t\right) \frac{d s d t}{t^{2}}, x \in \mathbb{R}^{d} .
$$

So $\mathcal{A}_{h_{0}}^{2}(f)=\sum_{i} A_{\xi_{i}}^{2}(f)$. From Theorem 4 and Lemma 1 one easily deduces that the set of points where $f$ is differentiable coincides up to sets of Lebesgue measure 0 , with the set of points $x \in \mathcal{U}$ for which there exists $\delta=\delta(x)>0$ such that both conditions (1.3) and $\mathcal{A}_{\delta}^{2}(f)(x)<\infty$ hold. From (c) of Theorem 4 one can easily deduce a characterization of Sobolev spaces in several variables in terms of the conical square function $\mathcal{A}$ which holds for any $1<p<\infty$. More concretely, if $f \in L^{p}\left(\mathbb{R}^{d}\right)$ then $f \in W^{1, p}\left(\mathbb{R}^{d}\right)$ if and only if $\mathcal{A}_{1}(f) \in L^{p}\left(\mathbb{R}^{d}\right)$. It would be interesting to compare this result with the beautiful characterization of Sobolev spaces given in [AMV].

We finally introduce another higher dimensional natural extension of the square function $A$ which describes differentiability at almost every point of a given set of the euclidean space. Let $f$ be a measurable function defined in an open set $\mathcal{U} \subset \mathbb{R}^{d}$. Let $S^{d-1}$ denote the unit sphere in $\mathbb{R}^{d}$ and let $\sigma$ be the normalized surface measure in $S^{d-1}$. Assume $f \in L_{\text {loc }}^{2}(\mathcal{U})$. Consider

$$
\mathbf{A}^{2}(f)(x, h)=\int_{S^{d-1}} A_{\xi}^{2}(f)(x, h) d \sigma(\xi), x \in \mathcal{U}, 0<h<1
$$

and $\mathbf{A}(f)(x)=A(f)(x, 0)$. Consider also the following averaged version of $\tilde{\Delta}_{\xi}$. Given a measurable subset $E \subset S^{d-1}$, consider

$$
\tilde{\Delta}(f)(x, h, E)=\int_{E} \tilde{\Delta}_{\xi}(f)(x, h) d \sigma(\xi), x \in \mathbb{R}^{d}, 0<h<1
$$

Theorem 5. (a) Let $\mathcal{U}$ be an open set of $\mathbb{R}^{d}$ and let $f \in L_{\text {loc }}^{2}(\mathcal{U})$. Consider the set $A=\{x \in$ $\mathcal{U}: f$ is differentiable at $x\}$ and the set $B$ of points $x \in \mathcal{U}$ such that $\mathbf{A}(f)(x)<\infty$ for which there exists $\delta=\delta(x)>0$ such that $\sup \left\{\left|\Delta_{2}(f)(x, h)\right|:|h|<\delta\right\}<\infty$. Then, the sets $A$ and $B$ can differ at most by a set of Lebesgue measure zero.

(b) There exists a constant $C=C(d)>0$ such that for any $f \in L_{\mathrm{loc}}^{2}\left(\mathbb{R}^{d}\right)$, for almost every point $x \in\left\{x \in \mathbb{R}^{d}: \mathbf{A}(f)(x)=\infty\right\}$ and for any measurable subset $E \subset S^{d-1}$, one has

$$
\limsup _{h \rightarrow 0} \frac{|\tilde{\Delta}(f)(x, h, E)|}{\sqrt{\mathbf{A}^{2}(f)(x, h) \ln \ln \mathbf{A}^{2}(f)(x, h)}} \leq C .
$$

Finally let us mention an easy consequence of Theorems 4 and 5 which is related to a classical result. Let $f$ be a function defined in an open subset $\mathcal{U} \subset \mathbb{R}^{d}$. Let $w$ be a function defined in 
$(0,1]$ such that for any $x \in \mathcal{U}$ and $h \in \mathbb{R}^{d}, 0<|h| \leq h_{0}=\min \left\{1, \operatorname{dist}\left(x, \mathbb{R}^{d} \backslash \mathcal{U}\right) / 2\right\}$, one has $|f(x+h)+f(x-h)-2 f(x)| \leq|h| w(|h|)$. Consider

$$
W(s)=\int_{s}^{1} w^{2}(t) \frac{d t}{t} \quad, 0<s<1 .
$$

If $w$ is increasing and $W(0)<\infty$, Stein and Zygmund proved that $f$ is differentiable at almost every point of $\mathcal{U}$. See [SZ2] or part (a) of Theorem 5. See also [Ma] and [DN]. If $W(0)=\infty$, part (b) of Theorem 4 gives that there exists a constant $C_{1}=C_{1}(d)$ only depending on the dimension such that for any $\xi \in \mathbb{R}^{d}$ with $|\xi|=1$, at almost every $x \in \mathcal{U}$ one has

$$
\limsup _{h \rightarrow 0} \frac{\left|\tilde{\Delta}_{\xi}(f)(x, h)\right|}{\sqrt{W(|h|) \ln \ln W(|h|)}} \leq C_{1} .
$$

The paper is organized as follows. Next Section is devoted to the discrete setting of dyadic martingales and to obtain the exponential inequalities relating the growth of a dyadic martingale and its quadratic variation. In Section 3 we consider the one dimensional continuous setting and obtain the subgaussian estimate (1.2) relating $\tilde{\Delta}(f)$ and $A(f)$ which is the main technical tool in the proof of Theorem 1. In Sections 4 and 5 we again use the results in the discrete setting to prove Theorems 2 and 3, respectively. In Section 6 we consider functions of several real variables and prove Theorems 4 and 5. Finally in Section 7 several natural questions closely related to our results are collected.

\section{The Discrete Setting}

For $1 \leq \rho \leq 4$ and $k=0,1,2, \ldots$, let $\mathcal{D}_{k}(\rho)$ be the collection of $\rho$-dyadic intervals of generation $k$ in $\mathbb{R}$ of the form $\left[j 2^{-k} \rho,(j+1) 2^{-k} \rho\right)$ where $j$ is an integer. Let $\mathcal{D}(\rho)=\bigcup_{k \geq 0} \mathcal{D}_{k}(\rho)$ be the collection of all $\rho$-dyadic intervals. For $x \in \mathbb{R}$ let $I_{k}^{(\rho)}(x)$ be the unique interval in $\mathcal{D}_{k}(\rho)$ which contains $x$. Also $|E|$ denotes the Lebesgue measure of the measurable set $E \subset \mathbb{R}$. A $\rho$-dyadic martingale is a sequence of locally integrable functions $S=\left\{S_{k}^{(\rho)}\right\}_{k}$ such that for any $k=0,1,2, \ldots$, the function $S_{k}^{(\rho)}$ is measurable with respect to the $\sigma$-algebra $\mathcal{F}_{k}$ generated by $\mathcal{D}_{k}(\rho)$ and the conditional expectation of $S_{k+1}$ respect to $\mathcal{F}_{k}$ is $S_{k}$. In other words, for any $k=0,1,2, \ldots$, the function $S_{k}^{(\rho)}$ is constant in each $\rho$-dyadic interval of $\mathcal{D}_{k}(\rho)$ and

$$
\int_{I}\left(S_{k+1}^{(\rho)}(x)-S_{k}^{(\rho)}(x)\right) d x=0
$$

for any $I \in \mathcal{D}_{k}(\rho)$. The truncated maximal function of the martingale $S$ is defined by

$$
M_{n}(S)(x)=\sup _{k \leq n}\left|S_{k}^{(\rho)}(x)\right|, \quad x \in \mathbb{R}, \quad n=1,2, \ldots
$$

The truncated quadratic variation of $S$ is defined by

$$
\langle S\rangle_{n}^{2}(x)=\sum_{k=1}^{n}\left(S_{k}^{(\rho)}(x)-S_{k-1}^{(\rho)}(x)\right)^{2}, \quad x \in \mathbb{R}, \quad n=1,2, \ldots
$$

It is well known that many properties on the asymptotic behavior of a martingale can be described in terms of the size of its quadratic variation. More concretely, the sets $\left\{x \in \mathbb{R}: \lim _{k \rightarrow \infty} S_{k}^{(\rho)}(x)\right.$ exists $\}$, 
$\left\{x \in \mathbb{R}: M_{\infty}(S)(x)<\infty\right\}$ and $\left\{x \in \mathbb{R}:\langle S\rangle_{\infty}(x)<\infty\right\}$ can only differ on a set of Lebesgue measure zero. See [BG1] or [BM, p. 64]. Also, fixed $0<p<\infty$ and $I \in \mathcal{D}(\rho)$, the maximal function $M_{\infty}(S)$ is in $L^{p}(I)$ if and only if $\langle S\rangle_{\infty}$ is in $L^{p}(I)$. See [BG1], BG2]. These results give comparisons between $M_{\infty}(S)$ and $\langle S\rangle_{\infty}$ on the sets where they are finite. In its complement, the following Law of the Iterated Logarithm governs the growth of the martingale,

$$
\limsup _{n \rightarrow \infty} \frac{\left|S_{n}^{(\rho)}(x)\right|}{\sqrt{\langle S\rangle_{n}^{2}(x) \ln \ln \langle S\rangle_{n}^{2}(x)}} \leq \sqrt{2}
$$

at almost every point $x \in\left\{x \in \mathbb{R}:\langle S\rangle_{\infty}(x)=\infty\right\}$. This result follows from good $\lambda$-inequalities, with subgaussian decay, which relate the growth of $M_{n}(S)$ and $\langle S\rangle_{n}$. See [St], [CWW] or [BM]. We start with a well known result in the same vein (see [BM, p. 47]) whose proof is included for the sake of completeness.

Lemma 2.1. Let $S=\left\{S_{k}^{(\rho)}\right\}$ be a $\rho$-dyadic martingale. Fix $I_{0} \in \mathcal{D}_{0}(\rho)$ and assume $S_{0}^{(\rho)} \equiv 0$ on $I_{0}$. Then

$$
\int_{I_{0}} \exp \left(S_{n}^{(\rho)}(x)-\frac{1}{2}\langle S\rangle_{n}^{2}(x)\right) d x \leq\left|I_{0}\right|, \quad n=1,2, \ldots
$$

Proof. Fix $I \in \mathcal{D}_{n-1}(\rho)$. Denote by $a_{n}(I)$ the constant value of $S_{n-1}^{(\rho)}-\frac{1}{2}\langle S\rangle_{n}^{2}$ on $I$. Then

$$
\int_{I} \exp \left(S_{n}^{(\rho)}(x)-\frac{1}{2}\langle S\rangle_{n}^{2}(x)\right) d x=\exp \left(a_{n}(I)\right)\left(\int_{I} \exp \left(S_{n}^{(\rho)}(x)-S_{n-1}^{(\rho)}(x)\right) d x\right) .
$$

Let $g=S_{n}^{(\rho)}-S_{n-1}^{(\rho)}$. Observe that $|g|$ has a constant value on $I$ which will be called $|g(I)|$. Since $\int_{I} g(x) d x=0$, using the elementary estimate $\cosh (x) \leq \exp \left(x^{2} / 2\right)$, we deduce

$$
\int_{I} \exp (g(x)) d x=|I| \cosh (|g(I)|) \leq|I| \exp \left(\frac{1}{2}|g(I)|^{2}\right) .
$$

Hence

$$
\int_{I} \exp \left(S_{n}^{(\rho)}(x)-\frac{1}{2}\langle S\rangle_{n}^{2}(x)\right) d x \leq \int_{I} \exp \left(S_{n-1}^{(\rho)}(x)-\frac{1}{2}\langle S\rangle_{n-1}^{2}(x)\right) d x .
$$

Adding over all $I \in \mathcal{D}_{n-1}(\rho)$ contained in $I_{0}$ we deduce

$$
\int_{I_{0}} \exp \left(S_{n}^{(\rho)}(x)-\frac{1}{2}\langle S\rangle_{n}^{2}(x)\right) d x \leq \int_{I_{0}} \exp \left(S_{n-1}^{(\rho)}(x)-\frac{1}{2}\langle S\rangle_{n-1}^{2}(x)\right) d x
$$

and the result follows.

We now easily deduce

Lemma 2.2. Let $S=\left\{S_{k}^{(\rho)}\right\}$ be a $\rho$-dyadic martingale. Fix $I_{0} \in \mathcal{D}_{0}(\rho)$ and assume $S_{0}^{(\rho)} \equiv 0$ on $I_{0}$. Then for any $n=1,2, \ldots$ and any $\lambda>0$ one has

$$
\left|\left\{x \in I_{0}: \sup _{k \leq n}\left(S_{k}^{(\rho)}(x)-\frac{1}{2}\langle S\rangle_{k}^{2}(x)\right)>\lambda\right\}\right| \leq e^{-\lambda}\left|I_{0}\right| .
$$


Proof. Fix $\lambda>0$. Fix the integer $n \geq 1$ and consider the stopping time $\tau(x)$ defined as the minimum between the indices $k \leq n$ for which $S_{k}(x)-\frac{1}{2}\langle S\rangle_{k}^{2}(x)>\lambda$, and $n$. Apply Lemma 2.1 to the stopped martingale $S^{\tau}$ defined as $S^{\tau}(x)=S_{\tau(x)}^{(\rho)}(x)$, to get

$$
\int_{I_{0}} \exp \left(S_{n}^{\tau}(x)-\frac{1}{2}\left\langle S^{\tau}\right\rangle_{n}^{2}(x)\right) d x \leq\left|I_{0}\right| \text {. }
$$

Since $S_{n}^{\tau}-\frac{1}{2}\left\langle S^{\tau}\right\rangle_{n}^{2}>\lambda$ on the set $\left.\left\{x \in I_{0}: \sup _{k \leq n}\left(S_{k}^{(\rho)}(x)-\frac{1}{2}\langle S\rangle_{k}^{2}(x)\right)>\lambda\right)\right\}$, the proof is completed.

Let $S=\left\{S_{k}^{(\rho)}\right\}$ be a $\rho$-dyadic martingale. For $n=1,2 \ldots$, consider $N_{n}=N_{n}(S)$ defined as

$$
N_{n}(x)=\left(\sup _{k \leq n}\left(S_{k}^{(\rho)}(x)-\frac{1}{2}\langle S\rangle_{k}^{2}(x)\right)\right)^{+} .
$$

Here $x^{+}=\max \{x, 0\}, x \in \mathbb{R}$. From Lemma 2.2 we easily deduce the following result.

Lemma 2.3. Let $S=\left\{S_{k}^{(\rho)}\right\}$ be a $\rho$-dyadic martingale. Fix $I_{0} \in \mathcal{D}_{0}(\rho)$ and assume $S_{0}^{(\rho)} \equiv 0$ on $I_{0}$. Then for any $0<\alpha<1$ and any integer $n \geq 1$, one has

$$
\int_{I_{0}} \exp \left(\alpha N_{n}(x)\right) d x \leq \frac{1}{1-\alpha}\left|I_{0}\right| .
$$

Proof. Fix the integer $n \geq 1$ and $0<\alpha<1$. Since

$$
\exp \left(\alpha N_{n}(x)\right) \leq 1+\exp \left(\alpha \sup _{k \leq n}\left(S_{k}^{(\rho)}(x)-\frac{1}{2}\langle S\rangle_{k}^{2}(x)\right)\right)
$$

we have

$$
\int_{I_{0}} \exp \left(\alpha N_{n}(x)\right) d x \leq\left|I_{0}\right|+\int_{I_{0}} \exp \left(\alpha \sup _{k \leq n}\left(S_{k}^{(\rho)}(x)-\frac{1}{2}\langle S\rangle_{k}^{2}(x)\right)\right) d x .
$$

By Lemma 2.2, the integral in the right hand side term is bounded by

$$
\alpha\left|I_{0}\right| \int_{0}^{\infty} e^{\alpha \lambda} e^{-\lambda} d \lambda=\frac{\alpha}{1-\alpha}\left|I_{0}\right|
$$

Let $S=\left\{S_{k}^{(\rho)}\right\}$ be a $\rho$-dyadic martingale. Fix $I_{0} \in \mathcal{D}_{0}(\rho)$. It is clear that for any $k \geq 1$, orthogonality gives that

$$
\int_{I_{0}}\left(S_{k}^{(\rho)}(x)-S_{0}^{(\rho)}(x)\right)^{2} d x=\int_{I_{0}}\langle S\rangle_{k}^{2}(x) d x
$$

We end this section with a local version of this result which will be used later.

Lemma 2.4. Let $S=\left\{S_{k}^{(\rho)}\right\}$ be a $\rho$-dyadic martingale. Fix $I_{0} \in \mathcal{D}_{0}(\rho)$ and assume $S_{0}^{(\rho)} \equiv 0$ on $I_{0}$. Consider the set $E=\left\{x \in I_{0}: \sup _{k}\left|S_{k}^{(\rho)}(x)\right| \leq 1\right\}$. Then there exists an absolute constant $C$, independent of $S$ and $I_{0}$, such that

$$
\int_{E}\langle S\rangle_{\infty}^{2}(x) d x \leq C
$$


Proof. Write $S_{k}=S_{k}^{(\rho)}$ and let $S_{k}(I)$ denote the constant value of $S_{k}$ in the interval $I \in \mathcal{D}_{k}(\rho)$. Let $\mathcal{G}$ be the family of maximal $\rho$-dyadic intervals $I$ such that $\left|S_{k}(I)\right|>1$. Here $k$ is the integer for which $I \in \mathcal{D}_{k}(\rho)$. It is clear that the set $E$ does not intersect the interior of any interval in $\mathcal{G}$. Let $\mathcal{G}_{1}$ be the subcollection of $\rho$-dyadic intervals $I \in \mathcal{G}$ for which $\left|S_{k}(I)\right|>10$, where again $I \in \mathcal{D}_{k}(\rho)$. We claim that if $I \in \mathcal{G}_{1}$ and $I^{\prime}$ is the $\rho$-dyadic brother of $I$, that is $\left|I^{\prime}\right|=|I|$ and $I \cup I^{\prime} \in \mathcal{D}(\rho)$, then $I^{\prime} \in \mathcal{G}$. Actually if $I^{*}=I \cup I^{\prime}$ is the $\rho$-dyadic father of $I$, by maximality, $I^{*}$ is not in $\mathcal{G}$, that is, $\left|S_{l}\left(I^{*}\right)\right| \leq 1$, where $I^{*} \in \mathcal{D}_{l}(\rho)$. Since $S_{l}\left(I^{*}\right)=\left(S_{l+1}(I)+S_{l+1}\left(I^{\prime}\right)\right) / 2$ and $\left|S_{l+1}(I)\right|>10$, we deduce that $\left|S_{l+1}\left(I^{\prime}\right)\right|>8$. Hence $I^{\prime} \in \mathcal{G}$ as claimed. So, $\rho$-dyadic brothers of intervals in $\mathcal{G}_{1}$ are in $\mathcal{G}$. Hence the interiors of $\rho$-dyadic fathers of intervals in $\mathcal{G}_{1}$ do not intersect $E$. Now, stop the martingale $S$ either at intervals which are $\rho$-dyadic fathers of intervals in $\mathcal{G}_{1}$ or at intervals in $\mathcal{G}$. Let $S^{\tau}$ be the corresponding stopped martingale and observe that $\left\|S^{\tau}\right\|_{\infty} \leq 10$. Since for any $x \in E$ one has $S_{k}(x)=S_{k}^{\tau}(x)$ for any $k$, we deduce that $\langle S\rangle_{\infty}(x)=\left\langle S^{\tau}\right\rangle_{\infty}(x)$ for any $x \in E$. Thus

$$
\int_{E}\langle S\rangle_{\infty}^{2}(x) d x=\int_{E}\left\langle S^{\tau}\right\rangle_{\infty}^{2}(x) d x \leq \int_{I_{0}}\left\langle S^{\tau}\right\rangle_{\infty}^{2}(x) d x=\int_{I_{0}}\left|S_{\infty}^{\tau}(x)\right|^{2} d x \leq 100 \rho .
$$

\section{The Law of the Iterated Logarithm}

Fix $1 \leq \rho<4$. Given a function $g$ defined in the real line, we denote by $S(g)$ the $\rho$-dyadic martingale $S(g)=\left\{S_{k}^{(\rho)}(g)\right\}_{k}$ defined as

$$
S_{k}^{(\rho)}(g)(x)=\frac{g(b)-g(a)}{b-a}, \quad k=0,1,2, \ldots,
$$

for $x \in I=[a, b) \in \mathcal{D}_{k}(\rho)$. Let $f$ be a function defined at almost every point $x \in \mathbb{R}$. Fixed $s \in \mathbb{R}$, consider the function $f_{s}$ defined by $f_{s}(x)=f(x-s), x \in \mathbb{R}$, and the $\rho$-martingale $S\left(f_{s}\right)=$ $\left\{S_{k}^{(\rho)}\left(f_{s}\right)\right\}_{k}$ which is well defined a.e. $(s, \rho) \in \mathbb{R} \times[1,4]$. Let $f_{E} f(x) d x$ denote the mean of a locally integrable function $f$ on the measurable set $E$, that is, $f_{E} f(x) d x=\left(\int_{E} f(x) d x\right) /|E|$. Next auxiliary result tells that the mean divided difference $\tilde{\Delta}(f)$ and the square function $A(f)$ defined in the Introduction, can be understood, respectively, as means of the martingales $S\left(f_{s}\right)$ and their quadratic variation.

Lemma 3.1. Let $f \in L_{\mathrm{loc}}^{1}(\mathbb{R})$. For $s \in \mathbb{R}$ consider the function $f_{s}$ defined by $f_{s}(x)=f(x-s)$, $x \in \mathbb{R}$, and the $\rho$-dyadic martingale $\left\{S_{k}^{(\rho)}\left(f_{s}\right)\right\}_{k}$ as defined in (3.1). For $0<y<2$ let $N=N(y)$ be the unique integer such that $H=H(y)=2^{N} y$ satisfies $1 \leq H<2$.

(a) For any $x \in \mathbb{R}$ and $0<y<2$, one has

$$
\tilde{\Delta}(f)(x, y)=\int_{y / 2}^{y} f_{x-h}^{x+h} \frac{f(s+h)-f(s-h)}{2 h} d s \frac{d h}{h}=\int_{H}^{2 H} f_{0}^{\rho} S_{N}^{(\rho)}\left(f_{s}\right)(x+s) d s \frac{d \rho}{\rho} .
$$

(b) Assume $f \in L_{\text {loc }}^{2}(\mathbb{R})$. Then for any $x \in \mathbb{R}$ and any $0<y<1$ one has

$$
\int_{y}^{H} f_{x-h}^{x+h} \Delta_{2}^{2}(f)(s, h) d s \frac{d h}{h}=\int_{H}^{2 H} f_{0}^{\rho}\left\langle S^{(\rho)}\left(f_{s}\right)\right\rangle_{N}^{2}(x+s) d s \frac{d \rho}{\rho} .
$$

Proof. (a) Fix $1 \leq \rho<4$ and $0<y<2$. An easy calculation shows

$$
f_{0}^{\rho} S_{N}^{(\rho)}\left(f_{s}\right)(x+s) d s=f_{0}^{2^{-N} \rho} \Delta(f)\left(x+t-2^{-N-1} \rho, 2^{-N-1} \rho\right) d t \quad, x \in \mathbb{R} .
$$


Integrating this identity with respect $d \rho / \rho$ and introducing the variables $s=x+t-2^{-N-1} \rho$, $h=2^{-N-1} \rho$, we deduce

$$
\int_{H}^{2 H} f_{0}^{\rho} S_{N}^{(\rho)}\left(f_{s}\right)(x+s) d s \frac{d \rho}{\rho}=\int_{y / 2}^{y} f_{x-h}^{x+h} \Delta(f)(s, h) d s \frac{d h}{h}
$$

which proves (a). To prove (b) fix $1 \leq \rho<4$ and observe that for any function $f$ defined in $[0, \rho]$ and any $k=0,1,2, \ldots$, one has

$$
\left(S_{k+1}^{(\rho)}(f)(x)-S_{k}^{(\rho)}(f)(x)\right)^{2}=\Delta_{2}^{2}(f)\left(\frac{a+b}{2}, 2^{-k-1} \rho\right)
$$

where $a=a(x), b=b(x)$ are defined by $x \in[a, b) \in \mathcal{D}_{k}(\rho)$. Using this identity, an easy calculation shows

$$
\begin{aligned}
& f_{0}^{\rho}\left(S_{k+1}^{(\rho)}\left(f_{s}\right)(x+s)-S_{k}^{(\rho)}\left(f_{s}\right)(x+s)\right)^{2} d s= \\
= & f_{0}^{2^{-k} \rho} \Delta_{2}^{2}(f)\left(x+t-2^{-k-1} \rho, 2^{-k-1} \rho\right) d t, \quad \text { a.e. } x \in \mathbb{R} .
\end{aligned}
$$

Integrating this identity with respect $d \rho / \rho$ and introducing the variable $h=2^{-k-1} \rho$, we deduce

$$
\begin{aligned}
& \int_{H}^{2 H} f_{0}^{\rho}\left(S_{k+1}^{(\rho)}\left(f_{s}\right)(x+s)-S_{k}^{(\rho)}\left(f_{s}\right)(x+s)\right)^{2} d s \frac{d \rho}{\rho}= \\
= & \int_{2^{-k-1} H}^{2^{-k} H} f_{0}^{2 h} \Delta_{2}^{2}(f)(x+t-h, h) d t \frac{d h}{h}, \quad \text { a.e. } x \in \mathbb{R} .
\end{aligned}
$$

Adding on $k=0, \ldots, N-1$, we deduce

$$
\int_{H}^{2 H} f_{0}^{\rho}\left\langle S^{(\rho)}\left(f_{s}\right)\right\rangle_{N}^{2}(x+s) d s \frac{d \rho}{\rho}=\int_{2^{-N} H}^{H} f_{0}^{2 h} \Delta_{2}^{2}(f)(x+t-h, h) d t \frac{d h}{h}, \quad x \in \mathbb{R} .
$$

Denote by $\tilde{A}^{2}(f)(x, y)$ the left term in the identity in part (b) of Lemma 3.1, that is

$$
\tilde{A}^{2}(f)(x, y)=\frac{1}{2} A^{2}(f)(x, y)+\int_{1}^{H} f_{x-h}^{x+h} \Delta_{2}^{2}(f)(s, h) d s \frac{d h}{h} \quad, x \in \mathbb{R}, 0<y<1
$$

Note that there exists an absolute constant $C>0$ such that

$$
\left|\tilde{A}^{2}(f)(x, y)-\frac{1}{2} A^{2}(f)(x, y)\right|<C \int_{x-2}^{x+2}|f(t)|^{2} d t
$$

For $f \in L_{\text {loc }}^{2}(\mathbb{R})$ and $0<h<1$, consider

$$
N(f)(x, h)=\frac{1}{\ln 4} \sup _{1 \geq y \geq h}\left(\tilde{\Delta}(f)(x, y)-\tilde{\Delta}(f)(x, H(y))-\frac{1}{2} \tilde{A}^{2}(f)(x, y)\right),
$$

where $H(y)$ is defined in Lemma 3.1. Recall that $1 \leq H(y)<2$. A version of Lemma 2.3 in the continuous setting is given in the following result. 
Lemma 3.2. Let $f \in L_{\mathrm{loc}}^{2}(\mathbb{R})$. For any $0<\alpha<1,0<h<1$ and any interval $I \subset \mathbb{R}$ with $|I|=1$ one has

$$
\int_{I} \exp (\alpha N(f)(x, h)) d x \leq \frac{C}{1-\alpha}
$$

where $C>0$ is a universal constant independent of $\alpha, h, I$ and $f$.

Proof. Fix $0<h<1$. For $h \leq y<1$, let $N(y)$ be the integer defined in the statement of Lemma 3.1, that is, $N(y)$ is the unique integer satisfying $H(y)=2^{N(y)} y \in[1,2)$. Lemma 3.1 gives that for any $x \in \mathbb{R}$ one has

$$
\begin{aligned}
\tilde{\Delta}(f)(x, y) & -\tilde{\Delta}(f)(x, H(y))-\frac{1}{2} \tilde{A}^{2}(f)(x, y) \\
& =\int_{H(y)}^{2 H(y)} f_{0}^{\rho}\left(S_{N(y)}^{(\rho)}\left(f_{s}\right)(x+s)-S_{0}^{(\rho)}\left(f_{s}\right)(x+s)-\frac{1}{2}\left\langle S^{(\rho)}\left(f_{s}\right)\right\rangle_{N(y)}^{2}(x+s)\right) d s \frac{d \rho}{\rho} .
\end{aligned}
$$

Since $y \geq h$ we have $N(y) \leq N(h)$ and we deduce

$$
N(f)(x, h) \leq \frac{1}{\ln 4} \int_{1}^{4} f_{0}^{\rho} N_{N(h)}^{(\rho)}(x+s) d s \frac{d \rho}{\rho},
$$

where

$$
N_{n}^{(\rho)}(x)=N_{n}^{(\rho)}\left(f_{s}\right)(x)=\sup _{k \leq n}\left(S_{k}^{(\rho)}\left(f_{s}\right)(x)-S_{0}^{(\rho)}\left(f_{s}\right)(x)-\frac{1}{2}\left\langle S^{(\rho)}\left(f_{s}\right)\right\rangle_{k}^{2}(x)\right)^{+}
$$

Fix $0<\alpha<1$. Jensen's inequality and Fubini Theorem give

$$
\int_{I} \exp (\alpha N(f)(x, h)) d x \leq \frac{1}{\ln 4} \int_{1}^{4} f_{0}^{\rho} \int_{I} \exp \left(\alpha N_{N(h)}^{(\rho)}(x+s)\right) d x d s \frac{d \rho}{\rho} .
$$

Lemma 2.3 gives that there exists a universal constant $C>0$ such that for every $s$ and $\rho$, one has

$$
\int_{I} \exp \left(\alpha N_{N(h)}^{(\rho)}(x+s)\right) d x \leq \frac{C}{1-\alpha}
$$

and the proof is completed.

The main technical step in the proof of our results is the following good $\lambda$-inequality with subgaussian decay.

Lemma 3.3. Let $f \in L_{\text {loc }}^{2}(\mathbb{R})$. For any $N, M>0$ with $M^{2}>4 N$ and any interval $I$ of unit length consider the set $E=E(M, N)$ of points $x \in I$ for which there exists $h=h(x)>0$ with $0<h<1$ such that

$$
\sup _{1 \geq y \geq h}(\tilde{\Delta}(f)(x, y)-\tilde{\Delta}(f)(x, H(y))) \geq M
$$

and

$$
\tilde{A}^{2}(f)(x, h) \leq N
$$

Then

$$
|E| \leq C \frac{M^{2}}{2 N} \exp \left(\frac{-M^{2}}{2 N \ln 4}\right) .
$$

Here $C$ is an absolute constant independent of $N, M, I$ and $f$. 
Proof. One can assume that there exists $h_{0}>0$ such that $h(x) \geq h_{0}>0$ for any $x \in E$. Observe that for any $\lambda>0$ and any $x \in E$ one has $(\ln 4) N(\lambda f)\left(x, h_{0}\right) \geq \lambda M-\lambda^{2} N / 2$. Lemma 3.2 gives that for any $0<\alpha<1$ one has

$$
|E| \exp \left(\alpha\left(\lambda M-\lambda^{2} N / 2\right) / \ln 4\right) \leq \frac{C}{1-\alpha} .
$$

Taking $\lambda=M / N$ one gets

$$
|E| \leq \frac{C \exp \left(\frac{-\alpha M^{2}}{2 N \ln 4}\right)}{1-\alpha} .
$$

The optimal choice $\alpha=1-2 N \ln 4 / M^{2}$ finishes the proof.

Using the subgaussian estimate of Lemma 3.3, an standard Borel-Cantelli argument gives the Law of the Iterated Logarithm stated in the Introduction as Theorem 1.

Proof of Theorem 1. By (3.2), in the statement $A^{2}(f)(x, h)$ can be replaced by $2 \tilde{A}^{2}(f)(x, h)$. Fix $R>1, L>1$ and $k=1,2, \ldots$ Consider the set $E_{k}$ of points $x \in[-L, L]$ for which there exists $h=h(x) \in(0,1)$ with $R^{k} \leq \tilde{A}^{2}(f)(x, h)<R^{k+1}$ and

$$
\tilde{\Delta}(f)(x, h)>R 2 \sqrt{(\ln 2) \tilde{A}^{2}(f)(x, h) \ln \ln \tilde{A}^{2}(f)(x, h)} .
$$

Since there exists an absolute constant $C>0$ such that

$$
|\tilde{\Delta}(f)(x, H(h))| \leq C \int_{x-2}^{x+2}|f(t)| d t,
$$

Lemma 3.3 applied with $N=R^{k+1}$ and $M=R \sqrt{4(\ln 2) R^{k} \ln \ln R^{k}}$ gives that for $k$ sufficiently large one has

$$
\left|E_{k}\right| \leq C(R)(\ln k) k^{-R}|L|
$$

where $C(R)$ denotes a constant depending on $R$. Thus $\sum\left|E_{k}\right|<\infty$ and we deduce

$$
\left|\bigcap_{m} \bigcup_{k>m} E_{k}\right|=0
$$

So, almost every point $x \in[-L, L]$ is at most, in a finite number of sets $E_{k}$. In particular for almost every $x \in\{x \in[-L, L]: A(f)(x)=\infty\}$ one has

$$
\tilde{\Delta}(f)(x, h)<R 2 \sqrt{(\ln 2) \tilde{A}^{2}(f)(x, h) \ln \ln \tilde{A}^{2}(f)(x, h)}
$$

if $h>0$ is sufficiently small. Since $L$ can be taken arbitrarily large, one deduces that

$$
\limsup _{h \rightarrow 0^{+}} \frac{\tilde{\Delta}(f)(x, h)}{\sqrt{\tilde{A}^{2}(f)(x, h) \ln \ln \tilde{A}^{2}(f)(x, h)}} \leq 2 R \sqrt{\ln 2}
$$

at almost every $x \in\{x \in \mathbb{R}: A(f)(x)=\infty\}$. Since the previous estimate also holds for $-f$ and any $R>1$, the proof is completed.

For future reference it is useful to state the following version of Lemma 3.3 . 
Lemma 3.4. Let $f \in L_{\mathrm{loc}}^{2}(\mathbb{R})$ and let $I \subset \mathbb{R}$ be an interval. For any $N, M>0$ with $M^{2}>4 N$, consider the set $E=E(M, N)$ of points $x \in I$ for which there exists $h=h(x)$ with $0<h<|I| / 2$ such that

$$
\sup _{|I|>y \geq h}(\tilde{\Delta}(f)(x, y)-\tilde{\Delta}(f)(x, H(y)|I|)) \geq M
$$

and

$$
\tilde{A}_{|I|}^{2}(f)(x, h)=\int_{h}^{H(h)|I|} \int_{x-t}^{x+t} \Delta_{2}^{2}(f)(s, t) \frac{d s d t}{t^{2}} \leq N .
$$

Then

$$
|E| \leq C \frac{M^{2}}{2 N} \exp \left(\frac{-M^{2}}{2 N \ln 4}\right)|I|
$$

Here $C$ is a universal constant.

\section{Sobolev Spaces}

In this Section we will show that Sobolev spaces can be described in terms of size conditions on the square function as stated in Theorem 3 of the Introduction. For $1<p<\infty$ let $W^{1, p}(\mathbb{R})$ be the Sobolev space of functions $f \in L^{p}(\mathbb{R})$ for which the distributional derivative $f^{\prime}$ is a function in $L^{p}(\mathbb{R})$. Equivalently, a function $f \in L^{p}(\mathbb{R})$ is in $W^{1, p}(\mathbb{R})$ if and only if

$$
\sup _{|h| \leq 1}\left|\frac{f(x+h)-f(x-h)}{2 h}\right| \in L^{p}(\mathbb{R}) .
$$

The necessity is clear because $|f(x+h)-f(x-h)| / 2|h|$ is bounded by the Hardy-Littlewood maximal function of $f^{\prime}$. The sufficiency can be proved as follows. There exists $h_{n} \rightarrow 0$ such that $h_{n}^{-1}\left(f\left(x+h_{n}\right)-f\left(x-h_{n}\right)\right)$ converges weakly in $L^{p}(\mathbb{R})$ to a certain function $g \in L^{p}(\mathbb{R})$. Then one may easily check that $g$ is the distributional derivative of $f$. Hence $f \in W^{1, p}(\mathbb{R})$.

We now prove Theorem 3 stated in the Introduction.

Proof of Theorem 3. Let $f \in W^{1, p}(\mathbb{R})$. In the case $2 \leq p<\infty$, a simple argument based in Lemma 3.1 will give that $A(f) \in L^{p}(\mathbb{R})$. Let $M\left(f^{\prime}\right)$ be the Hardy-Littlewood maximal function of $f^{\prime} \in L^{p}(\mathbb{R})$. Since for any $\rho \in[1,4]$ and any $s \in[0, \rho]$ we have

$$
M\left(S_{k}^{(\rho)}\left(f_{s}\right)\right)(x+s) \leq M\left(f^{\prime}\right)(x), \quad x \in \mathbb{R}
$$

we deduce that $M\left(S_{k}^{(\rho)}\left(f_{s}\right)\right) \in L^{p}(\mathbb{R})$ and $\left\|M\left(S_{k}^{(\rho)}\left(f_{s}\right)\right)\right\|_{p} \leq C_{1}(p)\left\|f^{\prime}\right\|_{p}$. Hence $\left\|\left\langle S^{(\rho)}\left(f_{s}\right)\right\rangle_{\infty}^{2}\right\|_{p / 2} \leq$ $C_{2}(p)\left\|f^{\prime}\right\|_{p}^{2}$. By Lemma 3.1, a.e. $x \in \mathbb{R}$ one has

$$
\frac{1}{2} A^{2}(f)(x) \leq \int_{1}^{4} f_{0}^{\rho}\left\langle S^{(\rho)}\left(f_{s}\right)\right\rangle_{\infty}^{2}(x+s) d s \frac{d \rho}{\rho} .
$$

Now, if $p \geq 2$, Minkowski inequality gives $\|A(f)\|_{p}^{2}=\left\|A^{2}(f)\right\|_{p / 2} \leq C_{3}(p)\left\|f^{\prime}\right\|_{p}^{2}$ and finishes the proof. In the case $1<p<2$, we will adapt an argument of Fefferman and Stein ([FS, p. 162]). Let $f \in W^{1, p}(\mathbb{R})$ and take $\lambda>0$. Consider the closed set $E=\left\{x \in \mathbb{R}: M\left(f^{\prime}\right)(x) \leq \lambda\right\}$. The main estimate of the proof is the following good- $\lambda$ inequality

$$
|\{x \in E: A(f)(x)>\lambda\}| \leq C|\mathbb{R} \backslash E|+\frac{C}{\lambda^{2}} \int_{0}^{\lambda} t\left|\left\{x \in \mathbb{R}: M\left(f^{\prime}\right)(x)>t\right\}\right| d t,
$$


where $C$ is a universal constant, independent of $f$ and $\lambda$. To prove (4.1) we will show that there exists an absolute constant $C_{1}>0$ such that for any $1 \leq \rho \leq 4$ and any $0 \leq s \leq \rho$, one has

$$
\int_{E}\left\langle S^{(\rho)}\left(f_{s}\right)\right\rangle_{\infty}^{2}(x+s) d x \leq C_{1} \lambda^{2}|\mathbb{R} \backslash E|+C_{1} \int_{0}^{\lambda} t\left|\left\{x \in \mathbb{R}: M\left(f^{\prime}\right)(x) \geq t\right\}\right| d t .
$$

Once (4.2) is proved, integrating on $\rho \in[1,4]$ and $s \in[0, \rho]$, Lemma 3.1 gives that

$$
\frac{1}{2} \int_{E} A^{2}(f)(x) d x \leq 4 C_{1} \lambda^{2}|\mathbb{R} \backslash E|+4 C_{1} \int_{0}^{\lambda} t\left|\left\{x \in \mathbb{R}: M\left(f^{\prime}\right)(x) \geq t\right\}\right| d t
$$

and (4.1) would follow taking $C=8 C_{1}$. To prove (4.2) fix $1 \leq \rho \leq 4$ and $0 \leq s \leq \rho$. Consider the family $G(\rho, s)$ of intervals of the form $\left[j 2^{-k} \rho-s,(j+1) 2^{-k} \rho-s\right)$ where $k \geq 0$ and $j$ are integers. In other words, intervals in $G(\rho, s)$ are translation of the $\rho$-dyadic intervals by $s$ units. Fix $I_{0} \in G(\rho, s)$ of length $\rho$, that is, of the form $I_{0}=[j \rho-s,(j+1) \rho-s)$ for some integer $j$. We may assume that $\left|I_{0} \backslash E \cap I_{0}\right|<1 / 2$. Consider the family $\mathcal{A}(\rho)=\mathcal{A}(\rho, \lambda, f)$ of maximal intervals in the family $G(\rho, s)$ contained in $I_{0} \backslash E$. Then

$$
\sum_{I \in \mathcal{A}(\rho)}|I|=\left|I_{0} \backslash E\right|
$$

Consider the martingale $\left\{S_{k}^{(\rho)}\left(f_{s}\right)(x+s)\right\}_{k}$ and stop it at the intervals of the family $\mathcal{A}(\rho)$. Let $S^{(\rho), \tau}$ be the corresponding stopped martingale. Orthogonality gives

$$
\int_{I_{0}}\left\langle S^{(\rho), \tau}\right\rangle_{\infty}^{2}(x+s) d x=\int_{I_{0}}\left(S_{\infty}^{(\rho), \tau}(x+s)-S_{0}^{(\rho), \tau}(x+s)\right)^{2} d x=A+B
$$

where

$$
\begin{aligned}
& A=\sum_{I \in \mathcal{A}(\rho)} \int_{I}\left(S_{k(I)}^{(\rho)}\left(f_{s}\right)(x+s)-S_{0}^{(\rho)}\left(f_{s}\right)(x+s)\right)^{2} d x, \\
& B=\int_{E \cap I_{0}}\left(S_{\infty}^{(\rho)}\left(f_{s}\right)(x+s)-S_{0}^{(\rho)}\left(f_{s}\right)(x+s)\right)^{2} d x .
\end{aligned}
$$

Here $k(I)$ is the integer satisfying $2^{-k(I)} \rho=|I|$. Fix $I \in \mathcal{A}(\rho)$. By maximality, its $(\rho, s)$-dyadic father $\tilde{I} \in G(\rho, s)$ contains a point $\tilde{x} \in E$. Hence $\int_{J}\left|f^{\prime}\right| \leq \lambda|J|$ for any interval $J$ containing $\tilde{x}$. Then $\int_{J}\left|f^{\prime}\right| \leq 3 \lambda|J|$ for any interval $J$ with $J \cap I \neq \emptyset$ and $|J| \geq|I|$. We deduce that $\left|S_{k(I)}^{(\rho)}\left(f_{s}\right)(x+s)\right|+\left|S_{0}^{(\rho)}\left(f_{s}\right)(x+s)\right| \leq 6 \lambda$ for any $x \in I$. Therefore (4.3) gives

$$
A \leq 36 \lambda^{2}\left|I_{0} \backslash E\right| .
$$

Consider $F(\rho, s)(t)=\left\{x \in E \cap I_{0}:\left|S_{\infty}^{(\rho)}\left(f_{s}\right)(x+s)-S_{0}^{(\rho)}\left(f_{s}\right)(x+s)\right|>t\right\}$. Since $\left|S_{\infty}^{(\rho)}\left(f_{s}\right)(x+s)\right|+$ $\left|S_{0}^{(\rho)}\left(f_{s}\right)(x+s)\right| \leq 2 M\left(f^{\prime}\right)(x) \leq 2 \lambda$ for $x \in E$, we have $F(\rho, s)(t) \subset\left\{x \in E \cap I_{0}: M\left(f^{\prime}\right)(x)>t / 2\right\}$ and

$$
B \leq 2 \int_{0}^{2 \lambda} t|F(\rho, s)(t)| d t \leq 2 \int_{0}^{2 \lambda} t\left|\left\{x \in E \cap I_{0}: M\left(f^{\prime}\right)(x) \geq t / 2\right\}\right| d t
$$

Since $S^{(\rho), \tau} \equiv S^{(\rho)}$ on $E \cap I_{0}$, identity (4.4) gives

$$
\int_{E \cap I_{0}}\left\langle S^{(\rho)}\left(f_{s}\right)\right\rangle_{\infty}^{2}(x+s) d x \leq 36 \lambda^{2}\left|I_{0} \backslash E\right|+8 \int_{0}^{\lambda} t\left|\left\{x \in I_{0}: M\left(f^{\prime}\right)(x) \geq t\right\}\right| d t .
$$


Adding this estimate over all $I_{0} \in G(\rho, s)$ of length $\rho$, estimate (4.2) follows. Thus (4.1) is proved. The rest of the proof is easy. From (4.1) it follows that

$$
\begin{gathered}
\int_{\mathbb{R}} A^{p}(f)(x) d x=p \int_{0}^{\infty} \lambda^{p-1}|\{x \in \mathbb{R}: A(f)(x)>\lambda\}| d \lambda \leq \\
\leq C p \int_{0}^{\infty} \lambda^{p-1}\left|\left\{x \in \mathbb{R}: M\left(f^{\prime}\right)(x) \geq \lambda\right\}\right| d \lambda+C p \int_{0}^{\infty} \lambda^{p-3} \int_{0}^{\lambda} t\left|\left\{x \in \mathbb{R}: M\left(f^{\prime}\right)(x) \geq t\right\}\right| d t d \lambda .
\end{gathered}
$$

Since $p<2$ each term is bounded by $C(p)\left\|M\left(f^{\prime}\right)\right\|_{p}^{p}$ and hence $\|A(f)\|_{p} \leq C_{1}(p)\left\|f^{\prime}\right\|_{p}$.

In the case $1<p \leq 2$ the converse follows easily from Lemma 3.1] Actually Holder's inequality gives that

$$
\int_{\mathbb{R}} \int_{1}^{2} \int_{0}^{\rho}\left\langle S^{(\rho)}\left(f_{s}\right)\right\rangle_{\infty}^{p}(x+s) \frac{d s d \rho}{\rho^{2}} d x \leq C(p) \int_{\mathbb{R}}\left(\int_{1}^{2} \int_{0}^{\rho}\left\langle S^{(\rho)}\left(f_{s}\right)\right\rangle_{\infty}^{2}(x+s) \frac{d s d \rho}{\rho^{2}}\right)^{p / 2} d x
$$

Now part (b) of Lemma 3.1, applied with $y=2^{-N}$ and letting $N \rightarrow \infty$, gives that

$$
\int_{\mathbb{R}} \int_{1}^{2} \int_{0}^{\rho}\left\langle S^{(\rho)}\left(f_{s}\right)\right\rangle_{\infty}^{p}(x+s) \frac{d s d \rho}{\rho^{2}} d x \leq C(p)\|A(f)\|_{p}^{p}
$$

Fubini's Theorem gives that almost every $\rho \in[1,2], s \in[0, \rho]$, the function $h_{s, \rho}$ defined as $h_{s, \rho}(x)=$ $\left\langle S^{(\rho)}\left(f_{s}\right)\right\rangle_{\infty}(x+s)$ is in $L^{p}(\mathbb{R})$ and one can choose $\rho$ and $s$ such that $\left\|h_{s, \rho}\right\|_{p} \leq C(p)\|A(f)\|_{p}$. Then the maximal function $M(x)=M_{\rho, s}(x)=\sup _{k}\left|S_{k}^{(\rho)}\left(f_{s}\right)(x+s)\right|$ is in $L^{p}(\mathbb{R})$ and the limit function $h$ defined by $h(x)=\lim _{k \rightarrow \infty} S_{k}^{(\rho)}\left(f_{s}\right)(x+s)$ is in $L^{p}(\mathbb{R})$. It is easy to see that $h$ is the distributional derivative of $f$ and hence $f \in W^{1, p}(\mathbb{R})$. Moreover $\left\|f^{\prime}\right\|_{p} \leq\|M\|_{p} \leq C(p)\|A(f)\|_{p}$.

Let us now consider the case $2 \leq p<\infty$. We first show that there exists a constant $C(p)>0$ such that for any $f \in W^{1, p}(\mathbb{R})$ one has

$$
\left\|f^{\prime}\right\|_{p} \leq C(p)\|A(f)\|_{p}
$$

Let $f \in W^{1, p}(\mathbb{R})$ and let $M\left(f^{\prime}\right)$ be the Hardy-Littlewood maximal function of $f^{\prime}$. Fixed $\lambda>0$ consider the open set $\mathcal{U}=\left\{x \in \mathbb{R}: M\left(f^{\prime}\right)(x)>\lambda\right\}$. Write $\mathcal{U}=\cup I_{j}$ where $\left\{I_{j}\right\}$ is a collection of pairwise disjoint open intervals. Since the end points of any $I_{j}$ are not in $\mathcal{U}$, we have that

$$
\int_{J}\left|f^{\prime}(x)\right| d x \leq \lambda|J|
$$

for any interval $J$ containing an end point of any $I_{j}$. Thus for any point $x \in I_{j}$, any $h \geq\left|I_{j}\right|$ and any $s \in(x-h, x+h)$ one has $|f(s+h)-f(s-h)|<2 \lambda h$. Hence $\left|\tilde{\Delta}(f)\left(x, H\left|I_{j}\right|\right)\right| \leq(\ln 2) \lambda$ for any $x \in I_{j}$ and any $1 \leq H \leq 2$. Fix $j$ and apply the subgaussian estimate of Lemma 3.4 to deduce that there exists a universal constant $C>0$ such that for any $0<\varepsilon<1$ one has

$$
\left|\left\{x \in I_{j}:\left|f^{\prime}(x)\right|>10 \lambda, A(f)(x) \leq \varepsilon \lambda\right\}\right| \leq C \exp \left(-1 / C \varepsilon^{2}\right)\left|I_{j}\right|
$$

Note that if $\left|f^{\prime}(x)\right|>10 \lambda$ then $x \in \mathcal{U}$. So, adding the previous estimate over $j$ one gets

$$
\left|\left\{x \in \mathbb{R}:\left|f^{\prime}(x)\right|>10 \lambda, A(f)(x) \leq \varepsilon \lambda\right\}\right| \leq C \exp \left(-1 / C \varepsilon^{2}\right)|\mathcal{U}|
$$

The rest of the proof of estimate (4.5) is standard. Write $\left\|f^{\prime}\right\|_{p}^{p} \leq A+B$ where

$$
\begin{aligned}
& A=p \int_{0}^{\infty} \lambda^{p-1}\left|\left\{x \in \mathbb{R}:\left|f^{\prime}(x)\right|>10 \lambda, A(f)(x) \leq \varepsilon \lambda\right\}\right| d \lambda, \\
& B=p \int_{0}^{\infty} \lambda^{p-1}|\{x \in \mathbb{R}: A(f)(x)>\varepsilon \lambda\}| d \lambda .
\end{aligned}
$$


Estimate (4.6) and the boundedness of the Hardy-Littlewood maximal function in $L^{p}$ give that there exists a constant $C_{1}(p)$ only depending on $p$ such that $A \leq C_{1}(p) \exp \left(-1 / C \varepsilon^{2}\right)\left\|f^{\prime}\right\|_{p}^{p}$. It is clear that $B \leq C(p, \varepsilon)\|A(f)\|_{p}^{p}$. We deduce that

$$
\left\|f^{\prime}\right\|_{p}^{p} \leq C_{1}(p) \exp \left(-1 / C \varepsilon^{2}\right)\left\|f^{\prime}\right\|_{p}^{p}+C(p, \varepsilon)\|A(f)\|_{p}^{p}
$$

Choosing $\varepsilon>0$ small enough so that $C_{1}(p) \exp \left(-1 / C \varepsilon^{2}\right)<1$, estimate (4.5) follows. The rest of the proof is now easy. Let $f \in L^{p}(\mathbb{R})$ with $A(f) \in L^{p}(\mathbb{R})$. Let $\varphi$ a smooth positive even function with $\|\varphi\|_{1}=1$. For $0<\varepsilon<1$ consider $\varphi_{\varepsilon}(x)=\varepsilon^{-1} \varphi(x / \varepsilon)$ and $f_{\varepsilon}=f * \varphi_{\varepsilon}$. Schwarz's inequality gives

$$
A^{2}\left(f_{\varepsilon}\right)(x) \leq\left(A^{2}(f) * \varphi_{\varepsilon}\right)(x), \quad x \in \mathbb{R} .
$$

If $p \geq 2$, Holder's inequality gives $\left\|A\left(f_{\varepsilon}\right)\right\|_{p} \leq\|A(f)\|_{p}$. Now estimate (4.5) gives that $\left\|\left(f * \varphi_{\varepsilon}\right)^{\prime}\right\|_{p} \leq$ $C(p)\|A(f)\|_{p}$ for any $0<\varepsilon<1$. We deduce that there exists a subsequence $\varepsilon_{n} \rightarrow 0$ such that $\left(f * \varphi_{\varepsilon_{n}}\right)^{\prime}$ converges weakly in $L^{p}(\mathbb{R})$ to a function $h \in L^{p}(\mathbb{R})$. It is easy to show that $h$ is the distributional derivative of $f$. Hence $f \in W^{1, p}(\mathbb{R})$. Moreover $\left\|f^{\prime}\right\|_{p}=\|h\|_{p} \leq C(p)\|A(f)\|_{p}$.

\section{Pointwise differentiability}

This Section is devoted to the proof of Theorem 2 .

Proof of Theorem 2. We first show that almost every point in $A$ is in $B$. If $f$ is differentiable at $x$, for $\varepsilon=\varepsilon(x)>0$ sufficiently small one has $\sup \left\{\left|\Delta_{2} f(x, h)\right|: 0<h<\varepsilon\right\}<\infty$. Using the notation of equation (3.1), consider the $\rho$-dyadic martingale $\left\{S_{k}^{(\rho)}\left(f_{s}\right)\right\}_{k}$ of the divided differences of the function $f_{s}$ defined as $f_{s}(t)=f(t-s), t \in \mathbb{R}$. It is clear that $A \subseteq \bigcup_{N=1}^{\infty} A_{N}$ where

$$
A_{N}=\left\{x \in \mathcal{U}: \sup _{s, \rho, k}\left|S_{k}^{(\rho)}\left(f_{s}\right)(x+s)\right| \leq N\right\} .
$$

Fix $N \geq 1$ and let $E \subset A_{N}$ be a bounded measurable set. Lemma 2.4 gives that for any $1 \leq \rho \leq 4$ and any $0 \leq s \leq \rho$, one has

$$
\int_{E}\left\langle S^{(\rho)}\left(f_{s}\right)\right\rangle_{\infty}^{2}(x+s) d x \leq C N^{2}|E|
$$

Integrating in $\rho \in[1,4]$ and $s \in[0, \rho]$, Lemma 3.1 yields

$$
\int_{E} A^{2}(f)(x) d x \leq C_{1} N^{2}|E|
$$

and hence $A(f)(x)<\infty$ a.e. $x \in E$. Hence almost every point of $A_{N}$ is in $B$.

Let us now show the opposite inclusion, that is, almost every point in $B$ is in $A$. Fix $N \geq 1$. It is sufficient to show that if $E$ is a bounded measurable set contained in

$$
\left\{x \in \mathcal{U}: A(f)(x) \leq N, \sup _{0<h<1 / N}\left|\Delta_{2} f(x, h)\right| \leq N\right\},
$$

then almost every point of $E$ is in $A$. For any $0<\delta<1$, there exists a subset $E(\delta) \subset E$ with $|E(\delta)|>(1-\delta)|E|$ and a constant $h_{0}=h_{0}(\delta)>0$ such that for any $x \in E(\delta)$ and any $0<h<h_{0}$ we have $|(x-h, x+h) \cap E| \geq h$. We want to show that for any $0<\delta<1$ almost 
every point of $E(\delta)$ is in $A$. Fix $\delta>0$. Denote by $\mathbf{1}_{\Gamma(x)}$ the characteristic function of the cone $\Gamma(x)=\left\{(s, t) \in \mathbb{R}_{+}^{2}:|s-x|<t<1\right\}$. We have

$$
\begin{aligned}
|E| N^{2} & \geq \int_{E} A^{2}(f)(x) d x=\int_{E} \int_{\mathbb{R}_{+}^{2}} \Delta_{2}^{2}(f)(s, t) \mathbf{1}_{\Gamma(x)}(s, t) \frac{d s d t}{t^{2}} d x \\
& \geq \int_{E(\delta) \times\left(0, h_{0}\right)} \Delta_{2}^{2}(f)(s, t)\left(\int_{E} \mathbf{1}_{\Gamma(x)}(s, t) d x\right) \frac{d t d s}{t^{2}} .
\end{aligned}
$$

Since for any $s \in E(\delta)$ and any $0<t<h_{0}$, the inner integral is bounded below by $t$, we deduce

$$
|E| N^{2} \geq \int_{E(\delta)} \int_{0}^{h_{0}} \Delta_{2}^{2}(f)(s, t) \frac{d t d s}{t} .
$$

In particular at almost every $s \in E(\delta)$ we have

$$
\int_{0}^{h_{0}} \Delta_{2}^{2}(f)(s, t) \frac{d t}{t}<\infty
$$

The classical result by Stein and Zygmund gives that $E(\delta) \subset A$ a.e. This finishes the proof.

\section{Several variables}

Given $\xi \in \mathbb{R}^{d}$ with $|\xi|=1$, let $\Pi(\xi)=\left\{x \in \mathbb{R}^{d}:\langle x, \xi\rangle=0\right\}$ be the hyperplane in $\mathbb{R}^{d}$ orthogonal to $\xi$ passing through the origin. For $x \in \mathbb{R}^{d}$ denote by $\tilde{x}$ its orthogonal projection onto $\Pi(\xi)$, that is, $x=\tilde{x}+\tilde{s} \xi$ where $\tilde{x} \in \Pi(\xi)$ and $\tilde{s} \in \mathbb{R}$. Let $f$ be a function defined in an open set $\mathcal{U} \subset \mathbb{R}^{d}$. For $\tilde{x} \in \Pi(\xi)$ consider the one variable function $f_{\tilde{x}}$ defined as $f_{\tilde{x}}(s)=f(\tilde{x}+s \xi)$ for $s \in\{s \in \mathbb{R}: \tilde{x}+s \xi \in \mathcal{U}\}$. Assume that $f$ is locally integrable and consider the mean divided difference in the direction of $\xi$, denoted by $\tilde{\Delta}_{\xi}(f)$, defined as $\tilde{\Delta}_{\xi}(f)(x, h)=\tilde{\Delta}\left(f_{\tilde{x}}\right)(\tilde{s}, h)$, where $x=\tilde{x}+\tilde{s} \xi$. In other words, for $x \in \mathcal{U}$ and $0<h<\operatorname{dist}\left(x, \mathbb{R}^{d} \backslash \mathcal{U}\right) / 2$,

$$
\tilde{\Delta}_{\xi}(f)(x, h)=\int_{h / 2}^{h} \int_{-t}^{t} \frac{f(x+s \xi+t \xi)-f(x+s \xi-t \xi)}{2 t} \frac{d s d t}{2 t^{2}}
$$

It is clear that if the ordinary directional derivative $D_{\xi}(f)(x)$ at the point $x \in \mathbb{R}^{d}$ exists, then $\tilde{\Delta}_{\xi}(f)(x, h)$ tends to $c D_{\xi}(f)(x)$ as $h$ tends to 0 . Here $c=\ln 2$. Similarly, if $f \in L_{\text {loc }}^{2}(\mathcal{U})$, its square function in the direction $\xi$ is denoted by $A_{\xi}(f)$ and defined by $A_{\xi}(f)(x, h)=A\left(f_{\tilde{x}}\right)(\tilde{s}, h)$, where $x=\tilde{x}+\tilde{s} \xi$. In other words, for $x \in \mathcal{U}$ and $0<h<h_{0}=\min \left\{1, \operatorname{dist}\left(x, \mathbb{R}^{d} \backslash \mathcal{U}\right) / 2\right\}$,

$$
A_{\xi}^{2}(f)(x, h)=\int_{h}^{h_{0}} \int_{-t}^{t}\left|\frac{f(x+s \xi+t \xi)+f(x+s \xi-t \xi)-2 f(x+s \xi)}{t}\right|^{2} \frac{d s d t}{t^{2}}
$$

As before we denote $A_{\xi}^{2}(f)(x)=A_{\xi}^{2}(f)(x, 0)$. We now prove Theorem 4 .

Proof of Theorem 4. As before write $x=\tilde{x}+\tilde{s} \xi$ where $\tilde{x} \in \Pi(\xi)$ and $\tilde{s} \in \mathbb{R}$. Consider the one variable function $f_{\tilde{x}}$ which is defined in an open set $\tilde{\mathcal{U}}=\tilde{\mathcal{U}}(\tilde{x}) \subset \mathbb{R}$. For any $\tilde{x} \in \Pi(\xi)$, the one dimensional result gives that the sets $\left\{s \in \tilde{\mathcal{U}}: f_{\tilde{x}}\right.$ is differentiable at $\left.s\right\}$ and

$$
\left\{s \in \tilde{\mathcal{U}}: A\left(f_{\tilde{x}}\right)(s)<\infty \text { and } \sup _{0<t<\varepsilon}\left|\frac{f_{\tilde{x}}(s+t)+f_{\tilde{x}}(s-t)-2 f_{\tilde{x}}(s)}{t}\right|<\infty \text { for some } \varepsilon=\varepsilon(\tilde{x}, s)>0\right\}
$$


can differ at most by a set of length zero. Hence by Fubini's Theorem part (a) follows. Similarly, the one variable result gives that for any $\tilde{x} \in \Pi(\xi)$ one has

$$
\limsup _{h \rightarrow 0} \frac{\left|\tilde{\Delta}\left(f_{\tilde{x}}\right)(s, h)\right|}{\sqrt{A^{2}\left(f_{\tilde{x}}\right)(s, h) \ln \ln A^{2}\left(f_{\tilde{x}}\right)(s, h)}} \leq \sqrt{2 \ln 2} .
$$

almost every $s \in\left\{s \in \tilde{\mathcal{U}}: A\left(f_{\tilde{x}}\right)(s)=\infty\right\}$. Part (b) follows again by Fubini's Theorem. Let us now prove part (c). As before for any $\tilde{x} \in \Pi(\xi)$ consider the function $f_{\tilde{x}}$. Let $m_{d-1}$ denote Lebesgue measure in $\Pi(\xi)$. Since $f \in L^{p}\left(\mathbb{R}^{d}\right)$ we have $f_{\tilde{x}} \in L^{p}(\mathbb{R})$ almost every $\left(m_{d-1}\right) \tilde{x} \in \Pi(\xi)$. Assume $D_{\xi} f \in L^{p}\left(\mathbb{R}^{d}\right)$. Fubini's Theorem gives that for almost every $\left(m_{d-1}\right) \tilde{x} \in \Pi(\xi)$, the function $f_{\tilde{x}}$ is absolutely continuous and $f_{\tilde{x}}^{\prime} \in L^{p}(\mathbb{R})$. Theorem 3 gives a constant $C>0$ such that for almost every $\left(m_{d-1}\right) \tilde{x} \in \Pi(\xi)$, one has

$$
C^{-1}\left\|A\left(f_{\tilde{x}}\right)\right\|_{L^{p}(\mathbb{R})} \leq\left\|f_{\tilde{x}}^{\prime}\right\|_{L^{p}(\mathbb{R})} \leq C\left\|A\left(f_{\tilde{x}}\right)\right\|_{L^{p}(\mathbb{R})}
$$

Integrating over $\tilde{x} \in \Pi(\xi)$ we deduce $C^{-1}\left\|A_{\xi}(f)\right\|_{p} \leq\left\|D_{\xi}(f)\right\|_{p} \leq C\left\|A_{\xi}(f)\right\|_{p}$. Conversely, assume $A_{\xi}(f) \in L^{p}\left(\mathbb{R}^{d}\right)$. Fubini's Theorem gives that for almost every $\left(m_{d-1}\right)$ point $\tilde{x} \in \Pi(\xi), A\left(f_{\tilde{x}}\right) \in$ $L^{p}(\mathbb{R})$. Theorem 3 gives that $f_{\tilde{x}} \in W^{1, p}(\mathbb{R})$. Hence $f$ is absolutely continuous along almost $\left(m_{d-1}\right)$ every line parallel to $\xi$ and its directional derivative in the sense of distributions is $f_{\tilde{x}}^{\prime}$. Moreover there exists a constant $C>0$ such that for almost every $\left(m_{d-1}\right) \tilde{x} \in \Pi(\xi)$, one has

$$
C^{-1}\left\|A\left(f_{\tilde{x}}\right)\right\|_{L^{p}(\mathbb{R})} \leq\left\|f_{\tilde{x}}^{\prime}\right\|_{L^{p}(\mathbb{R})} \leq C\left\|A\left(f_{\tilde{x}}\right)\right\|_{L^{p}(\mathbb{R})}
$$

Integrating over $\tilde{x} \in \Pi(\xi)$ we deduce $D_{\xi} f \in L^{p}\left(\mathbb{R}^{d}\right)$.

Let $f \in L_{\text {loc }}^{2}\left(\mathbb{R}^{d}\right)$. Let $S^{d-1}$ denote the unit sphere in $\mathbb{R}^{d}$ and let $\sigma$ be the normalized surface measure in $S^{d-1}$. As explained in the Introduction, we consider

$$
\mathbf{A}^{2}(f)(x, h)=\int_{S^{d-1}} A_{\xi}^{2}(f)(x, h) d \sigma(\xi) \quad, x \in \mathbb{R}^{d}, 0<h<1
$$

Denote by $e(z, x)=(z-x) /\|z-x\|$. An easy calculation shows

$$
\mathbf{A}^{2}(f)(x, h)=\int_{\Gamma(x) \cap\{t>h\}}\left|\frac{f(z+t e(z, x))+f(z-t e(z, x))-2 f(z)}{t}\right|^{2} \frac{d m(z)}{\|z-x\|^{d-1}} \frac{d t}{t^{2}}
$$

Here $\Gamma(x)=\left\{(z, t) \in \mathbb{R}^{d+1}: z \in \mathbb{R}^{d}, 0<t<1,|z-x|<t\right\}$ and $d m(z)$ denotes Lebesgue measure in $\mathbb{R}^{d}$. Consider also the following averaged version of $\tilde{\Delta}_{\xi}$. Given a measurable subset $E \subset S^{d-1}$, consider

$$
\tilde{\Delta}(f)(x, h, E)=\int_{E} \tilde{\Delta}_{\xi}(f)(x, h) d \sigma(\xi) \quad, x \in \mathbb{R}^{d}, 0<h<1
$$

An easy calculation shows

$$
\tilde{\Delta}(f)(x, h, E)=\int_{h / 2}^{h} \int_{E(t)} \frac{f(z+t e(z, x))-f(z-t e(z, x))}{2 t} \frac{d m(z)}{\|z-x\|^{d-1}} \frac{d t}{2 t^{2}}
$$

where $E(t)=\left\{z \in \mathbb{R}^{d}: 0<\|z-x\|<t, e(z, x) \in E\right\}$. The rest of this Section is devoted to the proof of Theorem 5 . We start with an elementary auxiliary result.

Lemma 6.1. Let $E$ be a measurable set contained in a ball $B \subset \mathbb{R}^{d}$. Assume $m(E)>2 m(B) / 3$. Then for any point $x \in B$ there exists $y \in E$ such that $(x+y) / 2 \in E$. 
Proof. Fist consider the one dimensional case $d=1$. One can assume $B=[0,1]$ and $x=0$. Since $|2 E \cap[0,1]|=2|E \cap[0,1 / 2]|>1 / 3$, we deduce that $|E \cap 2 E|>0$. So we may pick $y \in E \cap 2 E$. In the higher dimensional case $d>1$, observe that given $x \in B$, there exists a line segment $L \subset B$ ending at $x$ such that the length of $L \cap E$ is bigger than $2 / 3|L|$. Now the one dimensional result can be applied to obtain $y \in L \cap E$ such that $(x+y) / 2 \in E$.

Proof of Lemma 1. For $N=1,2, \ldots$, let $E_{N}$ be the set of points $x \in \mathcal{U}$ such that $\left|f\left(x+t e_{i}\right)-f(x)\right|<$ $N|t|$ for any $|t|<1 / N$ and $i=1,2, \ldots, d$ and moreover $\left|\Delta_{2}(f)(x, h)\right|<N$ for any $h \in \mathbb{R}^{d}$ with $0<|h|<1 / N$. Fix $N=1,2, \ldots$ and let us show that $f$ is differentiable at almost every point of $E_{N}$. Let $x$ be a point of density of $E_{N}$. Pick $\delta>0$ such that $m(E \cap B(x, t))>2 m(B(x, t)) / 3$ for any $0<t<\delta$. Here $B(x, t)$ denotes the ball centered at $x \in \mathbb{R}^{d}$ and radius $t>0$. We can assume that $\delta<1 / 2 N$. Let $h \in \mathbb{R}^{d}$ with $|h|<\delta$. Write $h=\sum_{j=1}^{d} h_{j} e_{j}, x_{0}=x$ and $x_{k}=x+\sum_{j=1}^{k} h_{j} e_{j}$ for $k=1,2, \ldots, d$. Then $f(x+h)-f(x)=\sum_{k=1}^{d}\left(f\left(x_{k}\right)-f\left(x_{k-1}\right)\right)$. Fix $k=1,2, \ldots, d$. Apply Lemma 6.1 to the point $x_{k} \in B(x, 2|h|)$ and the set $E_{N} \cap B(x, 2|h|)$ to obtain a point $y_{k} \in E_{N} \cap B(x, 2|h|)$ such that $\left(x_{k}+y_{k}\right) / 2 \in E_{N} \cap B(x, 2|h|)$. Note that $x_{k}=x_{k-1}+h_{k} e_{k}$. Observe that

$$
\begin{aligned}
& \frac{f\left(x_{k}\right)-f\left(x_{k-1}\right)}{|h|}-\frac{f\left(y_{k}+h_{k} e_{k}\right)-f\left(y_{k}\right)}{|h|}= \\
& =\frac{f\left(x_{k}\right)+f\left(y_{k}\right)-2 f\left(\left(x_{k}+y_{k}\right) / 2\right)}{|h|}-\frac{f\left(x_{k-1}\right)+f\left(y_{k}+h_{k} e_{k}\right)-2 f\left(\left(x_{k}+y_{k}\right) / 2\right)}{|h|}
\end{aligned}
$$

Since $\left(x_{k}+y_{k}\right) / 2 \in E_{N}$, the second term in the identity above is bounded by $2 N$. Since $y_{k} \in E_{N}$, we deduce that $\left|f\left(x_{k}\right)-f\left(x_{k-1}\right)\right|<3 N|h|$. Adding in $k=1,2, \ldots, d$, one deduces $|f(x+h)-f(x)|<$ $3 N d|h|$. We can now apply Stepanov Theorem to deduce that $f$ is differentiable at almost every point of $E_{N}$.

We now prove Theorem 5 .

Proof of Theorem 5. We start with part (a). For $N=1,2, \ldots$, consider the set $A_{N}$ of points $x \in \mathcal{U}$ such that $|f(x+h)-f(x)|<N|h|$ for any $h \in \mathbb{R}^{d}$ with $|h|<1 / N$. Note that every point of $A$ is in infinitely many $A_{N}$. Fix $N=1,2, \ldots$ and a bounded measurable set $E \subset A_{N}$, we will show that almost every point of $E$ is in $B$. Fix $\xi \in \mathbb{R}^{d}$ with $|\xi|=1$ and consider the orthogonal hyperplane $\Pi(\xi)=\left\{x \in \mathbb{R}^{d}:\langle x, \xi\rangle=0\right\}$. As before, for any $\tilde{x} \in \Pi(\xi)$ consider the function $f_{\tilde{x}}$ which is defined on the open one dimensional set $E(\tilde{x})=\{s \in \mathbb{R}: \tilde{x}+s \xi \in E\}$. Let $m_{d-1}$ denote Lebesgue measure in $\Pi(\xi)$. We have

$$
\int_{E} A_{\xi}^{2}(f)(x) d m(x)=\int_{\Pi(\xi)} \int_{E(\tilde{x})} A^{2}\left(f_{\tilde{x}}\right)(s) d s d m_{d-1}(\tilde{x})
$$

Since $E \subset A_{N}$, for any $\tilde{x} \in \Pi(\xi)$, the function $f_{\tilde{x}}$ is locally Lispchitz at each point of $E(\tilde{x})$ with constant $N$. The proof of Theorem 2 gives that there exists a constant $C>0$ independent of $\tilde{x}$ such that

$$
\int_{E(\tilde{x})} A^{2}\left(f_{\tilde{x}}\right)(s) d s<C N^{2}
$$

Hence

$$
\int_{E} A_{\xi}^{2}(f)(x) d m(x)<C(E) N^{2}
$$

Integrating on $\xi \in S^{d-1}$, we deduce that

$$
\int_{E} \mathbf{A}^{2}(f)(x) d m(x)<C(E) N^{2}
$$


and thus $\mathbf{A}(f)(x)<\infty$ at almost every $x \in E$. Hence almost every point of $E$ is in $B$. This finishes the first inclusion. To show the converse, for $N=1,2, \ldots$, consider the set $B_{N}$ of points $x \in \mathcal{U}$ such that $\mathbf{A}(f)(x)<N$ and $\left|\Delta_{2}(f)(x, h)\right|<N$ for any $h \in \mathbb{R}^{d}$ with $0<|h|<1 / N$. Observe that every point of $B$ is in infinitely many $B_{N}$. Fix $N=1,2, \ldots$ and a bounded measurable set $E \subset B_{N}$. We will show that $f$ is differentiable at almost every point of $E$. Since

$$
N^{2} m(E)>\int_{E} \mathbf{A}^{2}(f)(x) d m(x)=\int_{S^{d-1}} \int_{E} A_{\xi}^{2}(f)(x) d m(x) d \sigma(\xi),
$$

we deduce that for almost every $(\sigma) \xi \in S^{d-1}$ we have that $A_{\xi}(f)(x)<\infty$ at almost every $x \in E$. Part (a) of Theorem 4 gives that for almost every $(\sigma) \xi \in S^{d-1}$, the directional derivative $D_{\xi}(f)(x)$ exists at almost every point $x \in E$. Pick a basis $\left\{\xi_{1}, \xi_{2}, \ldots, \xi_{d}\right\} \in S^{d-1}$ such that for any $i=1,2, \ldots, d$, the corresponding directional derivative $D_{\xi_{i}}(f)(x)$ exists at almost every $x \in E$. Applying Lemma 1 one concludes that $f$ is differentiable at almost every point $x \in E$.

The proof of part (b) follows closely the arguments of the proof of Theorem 1. Let $f \in L_{\mathrm{loc}}^{2}\left(\mathbb{R}^{d}\right)$. Fix a measurable set $E \subset S^{d-1}$ with $\sigma(E)>0$ and consider the maximal function

$$
\mathbf{N}(f)(x, h)=\frac{1}{\sigma(E) \ln 4} \sup _{1 \geq y \geq h}\left(\tilde{\Delta}(f)(x, y, E)-\tilde{\Delta}(f)(x, H(y), E)-\frac{1}{2} \mathbf{A}^{2}(f)(x, y)\right)
$$

Since both $\tilde{\Delta}(f)(x, y, E)$ and $\mathbf{A}^{2}(f)(x, y)$ are means of their one dimensional analogues, Lemma 3.2 and Jensen's inequality give that for any cube $Q \subset \mathbb{R}^{d}$ with $m(Q)=1$ and any $0<\alpha<1$, $0<h<1$, one has

$$
\int_{Q} \exp (\alpha \mathbf{N}(f)(x, h)) d m(x) \leq \frac{C}{1-\alpha}
$$

where $C>0$ is a universal constant independent of $\alpha, h, Q$ and $f$. Now the proof proceeds as the proof of Theorem 1.

\section{Open Questions}

In this Section we collect several natural questions closely related to our results.

1. An easy calculation shows that

$$
\tilde{\Delta}(f)(x, h)=\frac{1}{4 h} \int_{x-2 h}^{x+2 h} \Delta(f)(s, 2 h) K((s-x) / h) d s \quad, x \in \mathbb{R}, 0<h<1,
$$

where $K$ is a function supported in $[-2,2], K \equiv 1$ in $[-1,1]$ and $K(s)=-1 / 3+4 / 3 w^{2}$ in $[-2,2] \backslash$ $[-1,1]$. It is natural to ask for a result similar to Theorem 1 for different kernels $K$. Also, consider

$$
\tilde{\Delta}^{*}(f)(x, h)=f_{x-h}^{x+h} \Delta f(s, h) d s
$$

Our arguments give a Law of the Iterated Logarithm relating the growth of $\tilde{\Delta}^{*}(f)\left(x, 2^{-N}\right)$ and a discrete version of $A(f)$ given by

$$
\sum_{k=1}^{N} \int_{x-2^{-k}}^{x+2^{-k}} \Delta_{2}^{2}(f)\left(s, 2^{-k}\right) d s
$$


2. It is natural to ask for a lower bound in the Law of the Iterated Logarithm given by Theorem 1 , More concretely, under which conditions on the function $f$ is the limsup in Theorem 1 bounded below by a positive constant? In the context of boundary behavior of harmonic functions in an upper half space, such lower bound was proved by Bañuelos, Klemes and Moore. See [BKM2] or [BM, p. 75]

3. Stein and Zygmund proved that the set of points where $f$ is differentiable in the $L^{2}$ sense coincides, up to sets of Lebesgue measure zero, with the set of points $x \in \mathbb{R}$ for which there exists $\delta=\delta(x)>0$ such that

$$
\int_{0}^{\delta} \Delta_{2}^{2}(f)(x, h) \frac{d h}{h}<\infty .
$$

See [SZ2] or [St1, p. 262]. So, it is natural to ask if this set also coincides almost everywhere with the set of points $x$ where $A(f)(x)<\infty$.

4. As in the classical situation, Theorem 2 applies to functions defined at every point of an open set. Let $f$ be a function defined in an open set $\mathcal{U}$. Given a set $E \subset \mathcal{U}$, it is natural to ask under which conditions the function $f$ coincides almost everywhere with a function which is differentiable in $E$. In one variable this was considered by Neugebauer $([\mathrm{N}])$ and his description was expressed in terms of the square function $g(f)$ mentioned in the Introduction. It is reasonable to expect a similar result with the square function $A(f)$ instead $g(f)$.

5. It is reasonable to expect that the set $A$ in Theorem 2 also coincides almost everywhere with the set

$$
C=\left\{x \in \mathcal{U}: \sup _{0<h<h_{0}} \int_{h / 2}^{h} \int_{x-y}^{x+y}\left|\frac{f(s+y)-f(s-y)}{y}\right|^{2} d s \frac{d y}{y^{2}}<\infty\right\}
$$

but we have not worked the details. It is obvious that $A \subseteq C$ but the converse is not clear and it could happen one has to add a pointwise condition on the symmetric differences.

6. In relation to Theorem 3, we mention that we have not explored analogue descriptions of Sobolev spaces with higher order derivatives.

7. We also do not know if Sobolev spaces $W^{1, p}\left(\mathbb{R}^{d}\right)$ can be described using the square function $\mathbf{A}(f)$ defined in (6.1). Let $f \in W^{1, p}\left(\mathbb{R}^{d}\right), 1<p<\infty$. Theorem 4 tells that for any $\xi \in \mathbb{R}^{d},|\xi|=1$, one has $A_{\xi}(f) \in L^{p}\left(\mathbb{R}^{d}\right)$ and $\left\|A_{\xi}(f)\right\|_{p}<C(p)\|f\|_{W^{1, p}\left(\mathbb{R}^{d}\right)}$. Minkowski integral inequality gives that $\|\mathbf{A}(f)\|_{p}<C(p)\|f\|_{W^{1, p}\left(\mathbb{R}^{d}\right)}$. The converse seem to require some work and we have not explored it.

\section{References}

[AMV] Alabern, R.; Mateu, J.; Verdera, J., A new characterization of Sobolev spaces on $\mathbb{R}^{n}$, Math. Ann. 354 (2012), no. 2, 589-626.

[AP] Anderson, J. M.; Pitt, L. D., Probabilistic behaviour of functions in the Zygmund spaces $\Lambda *$ and $\lambda *$, Proc. London Math. Soc. (3) 59 (1989), no. 3, 558-592.

[BKM1] Bañuelos, R; Klemes, I.; Moore, Ch. M., An analogue for harmonic functions of Kolmogorov's law of the iterated logarithm, Duke Math. J. 57 (1988), no. 1, 37-68.

[BKM2] Bañuelos, R; Klemes, I.; Moore, Ch. M., The lower bound in the law of the iterated logarithm for harmonic functions, Duke Math. J. 60 (1990), no. 3, 689-715.

[BM] Bañuelos, R.; Moore, Ch. N., "Probabilistic behavior of harmonic functions", Progress in Mathematics 175, Birkhäuser Verlag, Basel, 1999. 
[BG1] Burkholder, D. L.; Gundy, R. F., Extrapolation and interpolation of quasilinear operators on martingales, Acta Math. 124 (1970), 249-304.

[BG2] Burkholder, D. L.; Gundy, D. L., Distribution function inequalities for the area integral, Studia Math. 44 (1972), 527-544.

[CWW] Chang, S. Y.; Wilson, J. M.; Wolff, T. H., Some weighted norm inequalities concerning the Schrödinger operator, Comment. Math. Helv. 60 (1985), 217-246.

[Dlin1] Donaire, J. J.; Llorente, J. G.; Nicolau, A., Differentiability of functions in the Zygmund class, Proc. London Math. Soc. (2014) 108 (1): 133-158.

[Dlin2] Donaire, J. J.; Llorente, J. G.; Nicolau, A., Boundary values of Harmonic Gradients and Differentiability of Weierstrass type functions, to appear in Revista Mat. Iberoamericana arxiv.org/pdf/1202.0147.

[DN] Doubtsov, E.; Nicolau, A., Symmetric and Zygmund measures in several variables, Ann. Inst. Fourier (Grenoble) 52 (2002), no. 1, 153-177.

[FS] Fefferman, C.; Stein, E. M., $H^{p}$ spaces of several variables, Acta Math. 129 (1972), $137-193$.

[GJ] Garnett, J. B.; Jones, P. W., BMO from dyadic BMO, Pacific J. Math. 99 (1982), no. 2, $351-371$.

[Ma] Makarov, N. G., Probability methods in the theory of conformal mappings, (Russian) Algebra i Analiz 1 (1989), 3-59; translation in Leningrad Math. J. 1 (1990), 1-56.

[N] Neugebauer, C. J., Differentiability almost everywhere, Proc. Amer. Math. Soc. 16 (1965), $1205-1210$.

[SV] Slavin, L.; Volberg, A., The $s$-function and the exponential integral, in: "Topics in harmonic analysis and ergodic theory", Contemp. Math. 444, Amer. Math. Soc., Providence, RI, 2007, pp. 215-228.

[St1] Stein, E. M., "Singular integrals and differentiability properties of functions", Princeton Mathematical Series 30, Princeton University Press, Princeton, N.J., 1970.

[SZ1] Stein, E. M.; Zygmund, A., Smoothness and differentiability of functions, Ann. Univ. Sci. Budapest, Eötvös Sect. Math. 3-4 (1960/1961), 295-307.

[SZ2] Stein, E. M.; Zygmund, A., On the differentiability of functions, Studia Math. 23 (1963/1964), 247-283.

[St] Stout, W. F., A martingale analogue of Kolmogorov's law of the iterated logarithm, Z. Wahrscheinlichkeitstheorie und Verw. Gebiete 15 (1970), 279-290.

[W] Weiss, M., The law of the iterated logarithm for lacunary trigonometric series, Trans. Amer. Math. Soc. 91 (1959), 444-469. 